\title{
Orbifold Subfactors from Hecke Algebras
}

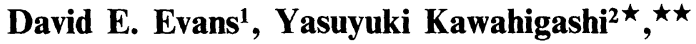 \\ ${ }^{1}$ Department of Mathematics, University College of Swansea, Singleton Park, Swansea, SA2 8PP \\ Wales, U.K. \\ 2 Department of Mathematics, University of California, Berkeley, CA 94720, USA. e-mail: \\ kawahiga@math.berkeley.edu
}

Received: 10 February 1992

\begin{abstract}
We apply the notion of orbifold models of $S U(N)$ solvable lattice models to the Hecke algebra subfactors of Wenzl and get a new series of subfactors. In order to distinguish our subfactors from those of Wenzl, we compute the principal graphs for both series of subfactors. An obstruction for flatness of connections arises in this orbifold procedure in the case $N=2$ and this eliminates the possibility of the Dynkin diagrams $D_{2 n+1}$, but we show that no such obstructions arise in the case $N=3$. Our tools are the paragroups of Ocneanu and solutions of Jimbo-MiwaOkado to the Yang-Baxter equation.
\end{abstract}

\section{Introduction}

A connection between solvable lattice models in statistical mechanics and subfactors in the theory of von Neumann algebras was soon realized after the pioneering work of Jones [Jo] on subfactors, see for example [EL]. Subsequently the theory of subfactors has had striking relations with knot theory, conformal field theory, quantum groups and so on (cf. [Ji, Kn, YG]). Recently the idea of an orbifold of a solvable lattice model has been studied by [DZ, FG, Ko], borrowing from the notion of an orbifold model in conformal field theory of [DHVW], who considered string propagation on toroidal orbifolds. In this paper we study the relation of subfactors to solvable lattice models, and apply the idea of orbifold lattice models to the subfactors of Wenzl [We] to get a new series of irreducible subfactors with the same index as the subfactors of Wenzl. Recall that his subfactors arise from representations of the Hecke algebra of type A and correspond to work of Jimbo-Miwa-Okado [JMO1, JMO2] in solvable lattice model theory, and also to the quantum groups $U_{q}\left(s l_{k}\right)$ and the 2-variable polynomial link invariant of [FYHLMO].

$\star$ Miller Research Fellow

$\star \star$ Current address: Department of Mathematical Sciences, University of Tokyo, Hongo, Tokyo, 113, JAPAN e-mail: yasuyuki@tansei.cc.u-tokyo.ac.jp 
The notion of orbifold models has also arisen recently in the structure theory of $C^{*}$-algebras. Here a striking new phenomenon has been discovered in the theory of $C^{*}$-algebras which are approximately finite dimensional and more generally in the larger class of $C^{*}$-algebras of real rank zero - which is probably a more accurate non-commutative analogue of a totally disconnected or zero dimensional space. For example, AF algebras have been constructed in a non-standard way as an inductive limit of homogeneous or subhomogeneous algebras on the circle (with careful choice of embeddings with the circle being wound around itself). The flip on the circle induces a symmetry on the algebra whose fixed point algebra is not AF (see [Bl, Ku and BEEK1-3] for generalisations to other groups). Similarly the flip on the interval has been exploited by [E11] to construct a symmetry on an AF algebra whose fixed point algebra is not even of real rank zero. The flip on the non-commutative 2torus $A_{\theta}$ [BEEK1-3] gives an AF fixed point algebra (a non-commutative toroidal orbifold - a non-commutative sphere with four singularities) [BK] see also [EE]. Taking the dual action one again gets a symmetry on the AF algebra $A_{\theta} \times \mathbf{Z}_{2}$ with fixed point algebra the non-AF irrational rotation algebra $A_{\theta}$. The message from this theory is that one can get something new and different from what one started with by considering the action of finite group on a system where there is a fixed point in the underlying basic structure. It is this same philosophy which we follow here to construct new subfactors.

The classification of subfactors of the approximately finite dimensional (AFD) factors is one of the most important and exciting problems in the theory of operator algebras. The tower of higher relative commutants has been a useful invariant for this problem. In particular, the Bratteli diagram of this tower is generated by iterating a graph (called the principal graph) beginning with some initial vertex. A. Ocneanu introduced a new notion paragroup as a combinatorial characterization of higher relative commutants in subfactor theory in [O1]. He announced in [O1] that a subfactor with finite index, trivial relative commutant, and "finite depth" can be generated by the tower of relative commutants, and thus they are classified by paragroups. Though his proof has not been presented yet, S. Popa gave a proof for this in [P3] without assuming the trivial relative commutant condition, and further announced necessary and sufficient conditions for such a generating property in [P4]. Thus a large class of subfactors of the AFD factor of type $\mathrm{II}_{1}$ can be classified by the paragroup approach.

A paragroup is a graph with a certain complex-valued function defined on squares arising from four edges of the graph. It can be regarded as a certain quantization of the Galois group. That is, instead of fields and subfields, we study factors and subfactors, which are non-commutative infinite dimensional $*$-algebras, and a graph is regarded as a generalization of the underlying set of a group. Group operations and duality are transformed into operations on the functions on squares.

Moreover, the study of paragroups can be regarded as differential geometry on finite graphs. Finite graphs are regarded as discrete models of compact manifolds, and the distinguished function on squares is an analogue of a connection. We call this function a connection, too. The key notion in the theory of Ocneanu is the analogue of flatness of connections.

Another aspect of paragroup theory is its connection with solvable lattice model theory like $[\mathrm{ABF}]$ (without a spectral parameter), and this is what we exploit in this paper. (See [DJMO, Ba] for solvable lattice model theory, for instance.) We have the following correspondences between these two theories. 
Table 0.1

\begin{tabular}{ll}
\hline Paragroups & Lattice models \\
\hline $\begin{array}{l}\text { Connections } \\
\text { Unitarity }\end{array}$ & Boltzmann weights \\
$\begin{array}{l}\text { Commuting square conditions } \\
\text { for towers of relative commutants }\end{array}$ & First inversion relations \\
Flatness of connections & Yrossing symmetry \\
\hline
\end{tabular}

More details on these correspondences are discussed in this paper. Note that the commuting square is a key notion in the approach of Jones to subfactors [P1, GHJ, Sect. 4.2]. We refer to [O1, O2, O3] for details of the theory of Ocneanu.

With his paragroup, A. Ocneanu announced classification of subfactors with index less than 4 in [O1]. In this case, the trivial relative commutant condition and the finite depth condition are satisfied automatically, so it is enough to classify paragroups with "order" less than 4. (The Jones index of a paragroup is an analogue of the order of a finite group.) In this case, the graphs we have (the "principal" graph as in [GHJ]) are among the Dynkin diagrams $A_{n}, D_{n}, E_{6}, E_{7}, E_{8}$.

The second author used the orbifold method in [Ka] to study subfactors with the principal graph $D_{n}$, the Dynkin diagrams, as "orbifolds" of subfactors of type $A_{n}$ by Jones [Jo]. An idea of "orbifold" is that if a paragroup has a certain symmetry, we can take its quotient by the symmetry. This idea was used in solvable lattice model theory by [FG, Ko], but the subfactor situation is more subtle as flatness is quite a strong requirement.

The Dynkin diagram $D_{n}$ can be regarded as an orbifold of $A_{2 n-3}$ by a $\mathbf{Z}_{2}$ symmetry. It is natural to apply the orbifold method also to Wenzl's subfactors, because Wenzl's subfactors are related to $S U(N)$ lattice models and the construction of Jones corresponds to the case $N=2$. Amongst the Dynkin diagrams, only the even $D_{2 n}$ appear as paragroups; the odd $D_{2 n+1}$ cannot appear as was announced in [O1] and proved in [Ka]. (Izumi gave a different proof of impossibility of $D_{2 n+1}$ independently in [I] based on Longo's sector theory [Ln1, Ln2], and its bimodule version was given by Sunder-Vijayarajan in [SV] independently. This approach was also claimed by Ocneanu without a proof.) This fact is interpreted in our orbifold setting as a $\mathbf{Z} / 2 \mathbf{Z}$-obstruction for flatness. In this paper, we show that in the case $N=3$, such an obstruction does not occur. The reason for this vanishing comes from the fact that 3 is odd while 2 is even. (Indeed, our method can be extended to all the odd prime $N$ with a slight modification.)

Our subfactors have the same index as those of Wenzl because the orbifold construction always gives the same Jones index as the original subfactor. We compute the principal graphs for both subfactors in order to distinguish them. For this purpose, we make use of the Yang-Baxter equation, or the star-triangle relation - the relation between flatness of connections and the Yang-Baxter equation is brought out in our discussion. Our approach also computes the paragroups of the subfactors of Wenzl, which he could not obtain. (He computed only the principal graphs with the $R$-matrix version of the Yang-Baxter equation in his unpublished work.)

During the preparation of this paper, the second author applied this orbifold method also to the classification of subfactors with the principal graph $D_{n}^{(1)}$ with M. Izumi in [IK]. These subfactors have index 4 and the extended Dynkin diagrams $D_{n}^{(1)}$ are regarded as orbifolds of $A_{2 n-5}^{(1)}$ by $\mathbf{Z}_{2}$-symmetry. 
Note that the procedures obtaining $D_{n}$ from $A_{2 n-3}$ and $D_{n}^{(1)}$ from $A_{2 n-5}^{(1)}$ by the $\mathbf{Z}_{2}$-symmetries are analogues of folding an interval and a circle by the $\mathbf{Z}_{2}$-flips in the $C^{*}$-algebra setting respectively.

The main part of this work was done while the first author was a visiting Professor at the Research Institute for Mathematical Sciences, Kyoto University. The authors thank the Research Institute for Mathematical Sciences at Kyoto University, University of Tokyo, and Hokkaido University for their hospitality. We also thank Professors T. Miwa and M. Okado for helpful conversations on solvable lattice models, Professor S. Popa for a comment related to Remark 5.9, and Professor H. Wenzl for a comment on his unpublished work in connection to Sect. 3.

\section{Lattice Models and AF Algebras}

One can associate to a graph $\Gamma$ a lattice model - a classical statistical mechanical model on a planar lattice $L$ in the following way. We first need to define the configuration space. We can take $L, \Gamma$ to be oriented, e.g., $L$ could be the two dimensional square lattice with downward orientation as in Fig. 1.1 and $\Gamma$ could be the $\mathscr{A}^{(n)}$ graph associated with the Weyl alcove of the level $k$ integrable representations of the Kac-Moody algebra $A_{N-1}^{(1)}=S U(N)^{\wedge}$ :

$$
P_{++}^{(n)}=\left\{\lambda=\sum_{i=1}^{N-1} \lambda_{i} \Lambda_{i} \mid \lambda_{i} \geqq 1, \sum_{i=1}^{N-1} \lambda_{i} \leqq n-1\right\},
$$

where the $\Lambda_{i}$ 's are the $N-1$ weights of the fundamental representations and $n=$ $k+N$.

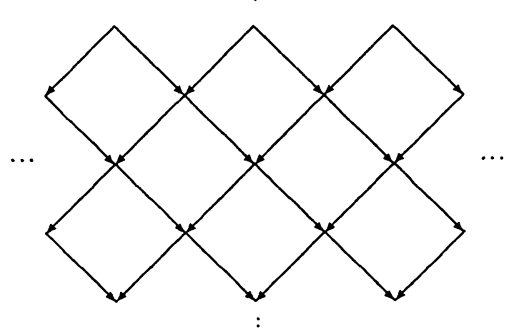

Fig. 1.1

For fixed $N$, we define $\mathscr{A}^{(n)}$ as follows. The vertices of $\mathscr{A}^{(n)}$ are given by elements of $P_{++}^{(n)}$ and its oriented edges are given by $N$ vectors $e_{i}$ defined by

$$
\begin{aligned}
e_{1} & =\Lambda_{1}, \\
e_{i} & =\Lambda_{i}-\Lambda_{i-1}, \quad i=1, \ldots, N-1, \\
e_{N} & =-\Lambda_{N-1} .
\end{aligned}
$$

That is, the adjacency matrix of the graph is given by $A_{\lambda, \mu}=\sum_{i=1}^{N} \delta_{\lambda+e_{i}, \mu}$. The following graph is an example for $N=3, n=6$. Note that the graphs are oriented. 


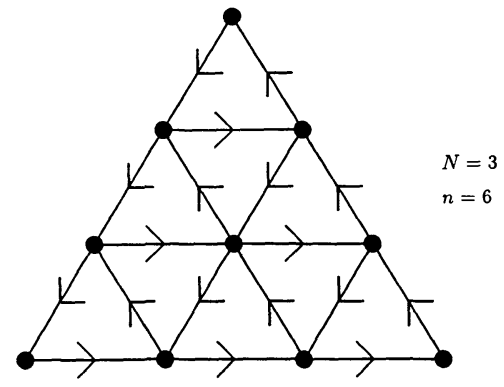

Fig. 1.2

A configuration is a map $\sigma: L^{(1)} \rightarrow \Gamma^{(1)}$ (associating an edge $\sigma_{\alpha}$ of $\Gamma$ to each edge $\alpha$ of $L$ ) which is compatible with the orientations of $L$ and $\Gamma$ (if they exist) and such that if $\alpha, \beta$ are incident edges of $L$, then $\sigma_{\alpha}$ and $\sigma_{\beta}$ are incident in $\Gamma$. Of course, we also have to specify the Hamiltonian or energy of a configuration. The energy associated to any face is a function of the shape of the boundary and the distribution of $\Gamma^{(1)}$ along it. The energy of a configuration $\sigma$ is then the sum of the energy of the faces. For the square lattice $L$ of Fig. 1.1, each face has a boundary of the form

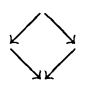

and the Boltzmann weight associated to a configuration

$$
\stackrel{\alpha}{\beta} \stackrel{\gamma}{\beta}
$$

where $\alpha \beta, \gamma \delta$ are paths of length two in $\Gamma$ with same initial and same terminal vertices could be denoted by $W(\alpha, \beta, \gamma, \delta)$. Strictly speaking, it could depend also on the position of the face in Fig. 1.1 in the lattice $L$. The Boltzmann weight of the configuration is then

$$
\prod_{\text {faces }} W(\alpha, \beta, \gamma, \delta)
$$

Writing

$$
W(\alpha, \beta, \gamma, \delta)=\stackrel{\alpha}{\alpha} \underset{\beta}{W} / \delta
$$

we see that a (translation invariant) energy function on $L$ for the graph $\Gamma$ is precisely the same as certain elements of the path $\mathrm{AF}$ algebra built from $\Gamma$.

The path algebra description of an AF algebra was introduced in [E1] or [E2], and was subsequently rediscovered by [O1, pages 128-129] or [O3, II.1], and Sunder [Su].

We define the path model of the AF algebra associated to a Bratteli diagram with standard embeddings. The AF algebra associated to a Bratteli diagram is unique (up to *-isomorphism) and any AF algebra is isomorphic to such an algebra with standard embeddings. We have a sequence $\Omega[m]$ of finite sets (each to represent the minimal central projections of a finite dimensional $C^{*}$-algebra $A_{n}$ ), and a multiplicity graph or matrix $\Lambda^{n}=\left[\lambda_{i j}^{(n)}\right]$ whose rows are labelled by $j \in \Omega[n]$ and columns by 
$i \in \Omega[n+1]$, where $\lambda_{i j}^{(n)}$ is the multiplicity of the simple algebra at vertex $i$ into that at vertex $j$. We adjoint $A_{0}=\mathbf{C}, \Omega[0]=\{*\}$, and only consider unital embeddings. The Bratteli diagram consists of the graph with vertices $\bigsqcup_{n \geqq 0} \Omega[n]$ and $\lambda_{j i}^{(n)}$ edges oriented between vertex $i$ in $\Omega[n]$ and $j$ in $\Omega[n+1]$ as indicated in Fig. 1.3.

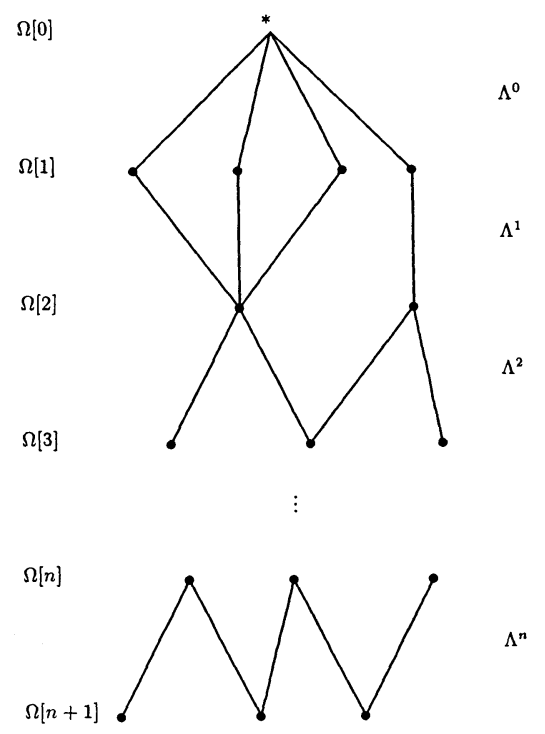

Fig. 1.3

The vertices $\Omega[n]$ denote the $n$-th level of the Bratteli diagram. For $m<n, i \in$ $\Omega[m], j \in \Omega[n]$, let Path $(i, j)$ denote the space of paths in the Bratteli diagram from $i$ to $j$. (Such paths have length $n-m$, since we have oriented the Bratteli diagram.) For $\mu \in$ Path $(i, j), i$ (respectively $j$ ) is called the initial (respectively terminal) vertex of $\mu$. Then let $\Omega[m, n]=\bigsqcup_{i \in \Omega[m], j \in \Omega[n]}$ Path $(i, j)$, the space of paths from level $m$ to level $n$. Let

$$
A_{i j}=\text { End } l^{2}(\operatorname{Path}(i, j))
$$

generated by matrix units $(\mu, v), \mu, v \in \operatorname{Path}(i, j)$, (called strings, denoted by String $_{m-n}^{i}(j)$ in the notation of [O3]), and

$$
A[m, n]=\oplus A_{i j}=\oplus \text { End } l^{2}(\operatorname{Path}(i, j)),
$$

where the summation is over all $i$ at level $m$, and $j$ at level $n$. If $[m, n] \subset\left[m^{\prime}, n^{\prime}\right]$, we embed $A[m, n]$ in $A\left[m^{\prime}, n^{\prime}\right]$ by

$$
(\mu, v) \mapsto \sum(\alpha \mu \beta, \alpha v \beta)
$$

for $\mu, v \in \operatorname{Path}(i, j)$, where the summation is over all $\alpha \in \operatorname{Path}\left(i^{\prime}, i\right), \beta \in \operatorname{Path}\left(j, j^{\prime}\right)$ and $i^{\prime}$ on level $m^{\prime}$ and $j^{\prime}$ on level $n^{\prime}$. Then

$$
A[0, m]^{\prime} \cap A[0, n]=A[m, n] .
$$


The AF algebra associated with the Bratteli diagram is then

$$
A=\underset{\lim }{\longrightarrow} A_{n},
$$

where $A_{n}=A[0, n]$ is embedded in $A_{n+1}$ by (1.7).

Example 1.1. Let $\Gamma$ be a locally finite graph, and $\Gamma_{0}^{(0)}$ a set of vertices from $\Gamma^{(0)}$. We can construct a Bratteli diagram $\left(\Gamma, \Gamma_{0}^{(0)}\right)^{\wedge}$ (and hence an AF algebra $\left.A\left(\Gamma, \Gamma_{0}^{(0)}\right)\right)$ as the space of semi-infinite paths beginning at $\Gamma_{0}^{(0)}$. Thus

$$
\left(\Gamma, \Gamma_{0}^{(0)}\right)^{\wedge}=\left\{\left(e_{n}\right)_{n=0}^{\infty}: e_{n} \in \Gamma^{(1)}, t\left(e_{n}\right)=i\left(e_{n+1}\right), i\left(e_{0}\right)=*, t\left(e_{0}\right) \in \Gamma_{0}^{(0)}\right\},
$$

where we have adjoined some vertex $*$ at the beginning of the diagram - which can sometimes be identified with a vertex of $\Gamma$.

A Boltzmann weight $W$ then gives an element $W_{n}$ in the path algebra $A[n-1, n+1]$ given by

$$
W_{n}=\sum_{\beta \searrow \beta^{\prime}}^{\alpha} \searrow_{\beta^{\prime}}^{\alpha^{\prime}}\left(\alpha \beta, \alpha^{\prime} \beta^{\prime}\right),
$$

where the summation is over all $\alpha \beta, \alpha^{\prime} \beta^{\prime} \in \Omega[n-1, n+1]$ with same initial and same terminal vertices.

Orbifold models have been considered in statistical mechanical models [FG, F], for $\mathbf{Z}_{2}$ and $\mathbf{Z}_{3}$ symmetries of the graphs in [DZ1, DZ2, and Z] as in Figs. 1.4 and 1.5 respectively, obtaining the orbifold graphs of Figs. 1.6 and 1.7 respectively.

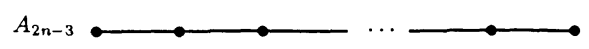

Fig. 1.4

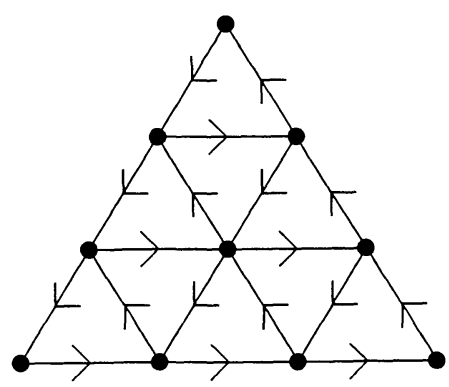

Fig. 1.5

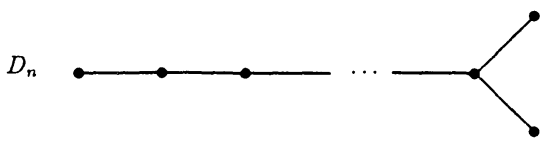

Fig. 1.6 


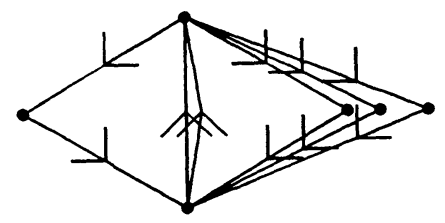

Fig. 1.7

The Boltzmann weights are given by $[\mathrm{FG}, \mathrm{F}]$,

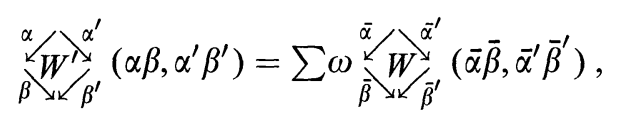

where the weights $W^{\prime}$ of the orbifold model are given a linear combination of those weights $W$ of the initial model, with the coefficient $\omega$ being square roots, and cubic roots, respectively (and vice versa, with the initial weights $W$ being expressed as a similar combination of the orbital weights $W^{\prime}$ ). Here we will interpret this orbifold construction via path algebras, in particular, this allows us to easily generalize the weights to the $\mathbf{Z}_{N}$ orbifolds of the $S U(N)$ models, and derive the Yang-Baxter equation without any pain. (The Yang-Baxter equation was checked by hand in the $N=2,3$ cases in [FG] and [F], whilst Di Francesco and Zuber [DZ1, p. 643] expected off critical orbifolds for $N>3$ models as consequences of identities between theta functions.)

Suppose that $G$ is a finite group of symmetries of the graph $\Gamma$ leaving a subset $\Gamma_{0}^{(0)}$ of the vertices $\Gamma^{(0)}$ globally invariant. Then there is an induced action of $G$ on strings $\alpha \mapsto g^{-1} \alpha$ and hence on the path algebra $A\left(\Gamma, \Gamma^{(0)}\right)_{0}$ by $(\alpha, \beta) \mapsto$ $\left(g^{-1} \alpha, g^{-1} \beta\right)$. The fixed point algebra $A\left(\Gamma, \Gamma_{0}^{(0)}\right)^{G}$ can be described as the path algebra built on an orbifold graph as follows.

For the case $\Gamma=\mathscr{A}^{(n)}$, we define an action of the cyclic group $\mathbf{Z}_{N}$ as follows. We set $A_{0}=*$, and label the other end vertices of the graph $\mathscr{A}^{(n)}$ by $A_{1}=A_{0}+(n-M) e_{1}, A_{2}=A_{1}+(n-N) e_{2}, \ldots, A_{N-1}=A_{N-2}+(n-N) e_{N-1}$. Define a rotation symmetry $\rho$ of the graph $\mathscr{A}^{(n)}$ by

$$
\rho\left(A_{j}+\sum_{k} c_{k} e_{k}\right)=A_{j+1}+\sum_{k} c_{k} e_{k+1},
$$

where the indices are in $\mathbf{Z} / N \mathbf{Z}$ and $c_{k} \in \mathbf{C}$. Note that $\rho^{N}=$ id. We take $\Gamma_{0}^{(0)}=$ $\left\{A_{0}, \ldots, A_{N-1}\right\}$, the orbit of $*$ under $\mathbf{Z}_{N}$.

In this case, the orbit of any vertex is either trivial or can be identified with the group $G$. Then at any level of the path algebra $A\left(\Gamma, \Gamma^{(0)}\right)$ we have either direct sums of matrix algebras $\bigoplus_{h \in G} A_{h}$, where $A_{h}$ is a matrix algebra transformed isomorphically to $A_{g+h}$ under the action of $g \in G$. To determine $\left[A\left(\Gamma, \Gamma_{0}^{(0)}\right)_{n}\right]^{G}$, note that the fixed point algebra of each $B$ is identified with a direct sum of a family $\left\{B_{h}: h \in G\right\}$ of isomorphic matrix algebras indexed by $G$, and the fixed point algebra of $\bigoplus_{h \in G} A_{h}$ is identified with $A_{0}$. Thus we can construct a graph $\Gamma / G$ to yield the Bratteli diagram of $A\left(\Gamma, \Gamma_{0}^{(0)}\right)$ as follows. We replace each trivial orbit in $\Gamma$ by a copy of $G$, and each non-trivial orbit in $\Gamma$ by a singleton. These are the vertices of the orbifold graph $\Gamma / G$. The edges of orbifold graph are obtained by 
considering the inclusion of $\left[A\left(\Gamma, \Gamma_{0}^{(0)}\right)_{n}\right]^{G}$ in $\left[A\left(\Gamma, \Gamma_{0}^{(0)}\right)_{n+1}\right]^{G}$. There are 3 cases to consider.

(a)
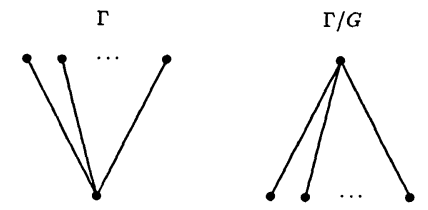

(b)
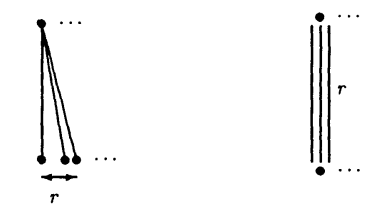

(c)
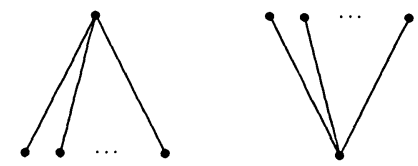

Fig. 1.8

In case (a), we have each vertex of a non-trivial orbit at level $n$ is joined to a trivial orbit by a single edge at level $n+1$ for $\Gamma$, which is reversed in the orbifold graph. Case (c) is similar. In case (b), we have each vertex of a non-trivial orbit at level $n$ is joined to $r$ vertices in a non-trivial orbit at level $n+1$ for $\Gamma$. In the orbifold graph, we have $r$ edges joining the trivial orbits.

This gives us the rules for computing the orbifold graph $\Gamma / G$, so that we have a filtered embedding $A(\Gamma / G, *) \rightarrow A\left(\Gamma, \Gamma_{0}^{(0)}\right)^{G}$ where $* \equiv \Gamma_{0}^{(0)} / G \in(\Gamma / G)^{(0)}$.

Te describe the embedding of the path algebra $A(\Gamma / G)$ in $A(\Gamma)$, it is useful to recall the cell calculus of Ocneanu [O1, O2, O3], which conveniently and concisely describe all filtered embeddings of path algebras.

First, we consider intertwiners between path algebras of sequences of graphs which have the same initial and same terminal vertices, in a similar fashion to how we constructed the path algebras themselves in the first place. If $\lambda_{1}, \lambda_{2}, \ldots$ and $\mu_{1}, \mu_{2}, \ldots$ are two sequences of graphs such that

$$
\begin{gathered}
t\left(\lambda_{n}\right)=i\left(\lambda_{n+1}\right), \quad t\left(\mu_{n}\right)=i\left(\mu_{n+1}\right), \\
i\left(\lambda_{n}\right)=i\left(\mu_{n}\right), \quad t\left(\lambda_{n}\right)=t\left(\mu_{n}\right),
\end{gathered}
$$

we can define $\left(\lambda_{r}, \mu_{r}\right)$ in

$$
\bigoplus_{i, j} \text { End }\left(l^{2} \operatorname{Path}_{\lambda_{r}}(i, j)\right), l^{2}\left(\operatorname{Path}_{\mu_{r}}(i, j)\right)
$$

to be the intertwiners between $\left(\lambda_{r}, \lambda_{r}\right) \equiv A(\lambda)[r, r+1]$ and $A(\mu)[r, r+1]$ generated by matrix units $(\mu, v)$, with $\mu \in \operatorname{Path}_{\lambda_{r}}(i, j), v \in \operatorname{Path}_{\mu_{r}}(i, j)$ with same initial and same terminal vertices $i$ and $j$ respectively. In particular, concatenating graphs, we have intertwiners

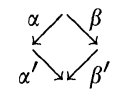


where $\alpha, \alpha^{\prime}, \beta, \beta^{\prime}$ are edges of $\lambda_{r}, \lambda_{r+1}, \mu_{r}, \mu_{r+1}$ respectively (where of course we do not really need (1.15) for $n=r$ ).

Consider a filtered $*$-homomorphism $\varphi$ between path algebras $A(\lambda)$ and $A(\mu)$ filtered in the sense that $A(\lambda)_{n}$ is taken to $A(\mu)_{n}$ with this restriction denoted by $\varphi_{n}$. Now the $*$-homomorphism $\varphi_{n}$ is up to an inner automorphism of $A(\mu)_{n}$ a path endomorphism of the type (1.7). More precisely, there is a graph $i_{n}$ between the $n$-th levels of $A(\lambda)$ and $A(\mu)$, and a unitary intertwiner

$$
u_{n} \in\left(i_{n} \lambda^{(n)} \ldots \lambda^{(0)}, \mu^{(n)} \ldots \mu^{(0)}\right)
$$

such that

$$
\varphi_{n}=\operatorname{Ad}\left(u_{n}\right) i_{n} .
$$

Then since $\mu^{(n)} \varphi_{n}=\varphi_{n+1} \lambda^{(n)}, w_{n+1}=j_{n}\left(u_{n}^{*}\right) u_{n+1}$ is an intertwiner in

$$
\left(i_{n+1} \lambda^{(n+1)} \ldots \lambda^{(0)}, \mu^{(n+1)} i_{n} \lambda^{(n)} \ldots \lambda^{(0)}\right)
$$

which commutes with

$$
\left(\lambda^{(n)} \cdots \lambda^{(0)}, \lambda^{(n)} \cdots \lambda^{(0)}\right)=A(\lambda)_{n} .
$$

Hence by the extension of (1.8) to intertwiners, $w_{n+1} \in\left(i_{n+1} \lambda^{(n+1)}, \mu^{(n+1)} i_{n}\right)$ and

implements $\varphi_{n}$ as in (1.17).

If all four graphs $i_{n}, \lambda_{n}, \mu_{n}, j_{n}$ are the same (as will be the case in the $\mathscr{A}^{(n)}$ models - see Fig. 1.2), then an intertwiner in $\left(\lambda_{n} j_{n}, i_{n} \mu_{n}\right)$ is also an element of the path algebra $\mathscr{A}(\lambda)$. In the theory of subfactors, the braid element plays a dual role. It may appear in the path algebra as a specialisation (see for example (2.5)) of a Boltzmann weight as in (1.11). There may also be a shift endomorphism $k$ (related to Kramers-Wannier duality [EL, CE]) of the path algebra, $k: \mathscr{A}(\lambda) \rightarrow \mathscr{A}(\lambda)$ taking the braid element

$$
\sigma_{i} \in \mathscr{A}[i-1, i+1] \rightarrow \sigma_{i+1} \in \mathscr{A}[i, i+2] .
$$

Indeed, because of the braid relation

$$
\sigma_{i} \sigma_{i+1} \sigma_{i}=\sigma_{i+1} \sigma_{i} \sigma_{i+1}
$$

we have

$$
\kappa\left(\sigma_{i}\right)=\lim _{n \rightarrow \infty} \sigma_{1} \sigma_{2} \cdots \sigma_{n}\left(\sigma_{i}\right) \sigma_{n}^{-1} \cdots \sigma_{2}^{-1} \sigma_{1}^{-1},
$$

and indeed we may have

$$
\kappa(x)=\lim _{n \rightarrow \infty} \sigma_{1} \sigma_{2} \cdots \sigma_{n}(x) \sigma_{n}^{-1} \cdots \sigma_{2}^{-1} \sigma_{1}^{-1}
$$


as in the $\mathscr{A}^{(n)}$ models (see [EG1, EG2] and [GW] for other examples). In this way, the braid elements appear as the intertwiners or connection for the filtered homomorphism $k$. Then when we use (1.17) to transform in the double complex picture of (2.1) an element $W$ such as a Boltzmann weight or a genuine partition function:

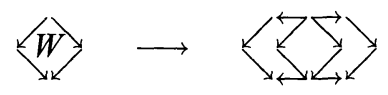

The elements

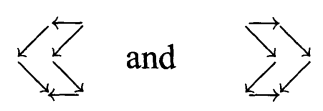

which appear here arise from "partition functions" of braid elements (with no spectral parameter). In this respect, the expressions

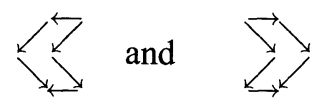

which we compute are not genuine partition functions but intertwiners between submodel and a model. Note that a unitary matrix cannot in any case have positive entries except in rather trivial cases. In this regard, the relation between connections and Boltzmann weights of Table 0.1 needs to be taken with a pinch of salt.

Before we complete the intertwiners between a path algebra and its orbifold model, let us recall the situation for subalgebras of UHF algebras arising as fixed point algebras of limit inner actions. (Of course, we could consider actions of AF algebras which involve both limit inner actions and graph (or Bratteli diagram) symmetries but we refrain from doing that since the extra notational complexity is not needed in our examples.)

Example 1.2. Let $\pi_{j}: G \rightarrow M_{n(j)}$, be a unitary implementation of a compact group $G$, and $\alpha_{g}=\bigotimes_{j=1}^{\infty} \operatorname{Ad} \pi_{j}$, the product type action of $G$ on the UHF algebra

$$
A=\bigotimes_{j=1}^{\infty} M_{n(j)}=\lim _{\rightarrow} A_{m},
$$

where $A_{m}=\bigotimes_{j=1}^{m} M_{n(j)}$. The Bratteli diagram of this tower of algebras is given by singletons $\Omega[m]$ and graphs $\mu^{(m)}$ with $n(m)$ edges connecting $\Omega[m]$ and $\Omega[m+1]$. The fixed point algebra $A^{G}$ is $\mathrm{AF}$ being the $C^{*}$-inductive limit of the fixed point algebras $A_{m}^{G}$.

Let $\left\{\chi_{\alpha}\right\}$ denote the irreducible characters of $G$, where $\chi_{0}$ is the trivial character, and $\chi^{(j)}$ the character of $\pi_{j}$. For each $m=0,1,2 \ldots$, let

$$
\chi^{(0)} \chi^{(1)} \cdots \chi^{(m)}=\sum \alpha_{m \alpha} \chi_{\alpha}
$$

be the decomposition of the character $\chi^{(0)} \cdots \chi^{(m)}$ corresponding to the representation $\bigotimes_{j=0}^{m} \pi_{n(j)}$ into irreducible characters where $a_{m \alpha}$ are positive integers. By Schur's lemma, the fixed point algebra $A_{m}^{G}$ has the following decomposition into simple components:

$$
A_{m}^{G} \cong \bigoplus_{\alpha} M_{a_{m \alpha}} .
$$


The multiplicity $\kappa_{\alpha \beta}^{(m)}$ of the embedding of the simple components $M_{a_{m \alpha}}$ into $M_{a_{m+1, \beta}}$ is determined by the decomposition of $\chi^{(m+1)} \chi_{\alpha}$ into irreducible characters

$$
\chi^{(m+1)} \chi_{\alpha}=\sum \kappa_{\alpha \beta}^{(m)} \chi_{\beta} .
$$

Thus $A^{G} \cong A(\kappa)$, but to understand the embedding $\varphi: \mathscr{A}(u) \rightarrow A(\mu)$, we need the Clebsch-Gordan coefficients which expresses the equality (1.22) at the representation level. Let $\Omega^{\prime}[m]$ denote the vertices at level $m$ of the Bratteli diagram of $\mathscr{A}(u)$, i.e., the irreducible representations of $G$ which figure in (1.22). Then define $i_{m}$ to be the graph with $\operatorname{dim} \pi_{\alpha}$ edges connecting $\alpha$ in $\Omega^{\prime}[m]$ to the singleton $\Omega[m]$. Then the connection or intertwiner describing the embedding of $\mathscr{A}(\kappa)$ in $\mathscr{A}(\mu)$ is the Clebsch-Gordan coefficient $\mathscr{U}$ in $\left(i_{m+1} \kappa^{(m)}, \lambda^{(m)} i_{m}\right)$ :

$$
\pi^{(m+1)} \otimes \pi_{\alpha} \cong \mathscr{U}\left(\bigoplus_{\beta} \pi \beta \otimes 1_{\kappa_{\alpha \beta}^{(m)}}\right) \mathscr{U}^{*}
$$

In a similar way, the connection for orbifold models is obtained as the ClebschGordan coefficients which put the cyclic permutations of Fig. 1.8 in diagonal form. If $\omega$ is a primitive $N^{\text {th }}$ root of unity, then

$$
\begin{aligned}
& \text { (a) } \begin{array}{lll}
1 & \rightarrow & n \\
& & \downarrow \\
m & \rightarrow & 1
\end{array}=\frac{1}{\sqrt{N}} \omega^{m-n}
\end{aligned}
$$

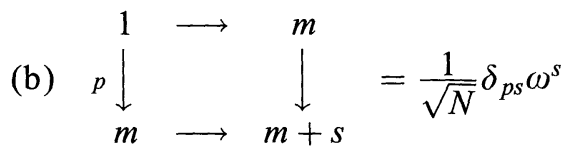

where $m, n \in \mathbf{Z}_{N}, p, s \in\{0,1,2, \ldots, r-1\} \subset \mathbf{Z}_{N}$.

The embedding $A^{G} \rightarrow A$ of limit inner actions of Example 1.2 or graph symmetries of Example 1.1 have the property that not only are they filtered but they also satisfy

$$
A[\lambda][m, n]^{G} \rightarrow A[\kappa][m, n] .
$$

To see this, note that the action of $G$ leaves invariant $A[\lambda][m, n]$ (e.g., by (1.8)) and

$$
A[\lambda][m, n]^{G} \subset A[\lambda][m, n] \cap A[\kappa][0, n]
$$

as $A[\kappa][0, n]=A[\lambda][0, n]^{G}$. Take $x \in A[\lambda][m, n]^{G}, y \in A[\kappa][0, m] \subset A[\lambda][0, m]$, then $x y=y x$ as $x \in A[\lambda][m, n], y \in A[\lambda][0, m]$. Hence $x \in A[\kappa][0, n] \cap A[\kappa][0, m]^{\prime}=$ $A[\kappa][m, n]$.

In particular, this means that if $W \in A[\lambda][m-1, m+1]^{G}$, then the corresponding $W^{\prime}$ of $A[\lambda][m-1, m+1]$ satisfy

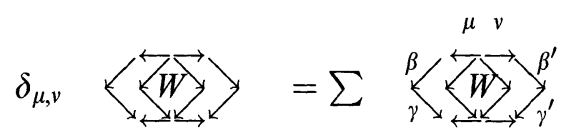


where

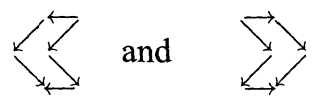

are the intertwining unitary connections. In the case of Example 1.1, this explains and verifies the vertex-IRF correspondence computations of [R, Appendix 4]. (Note also that Property $\mathrm{T}$ of $[\mathrm{R}]$ holds since we clearly have commuting squares as the conditional expectation $E_{n}: A_{n} \rightarrow A_{n}^{G}$ is consistently given by $E=\int \alpha_{g} d g$ ). Also it verifies the corresponding equation in the orbifold model Example 1.1 and explains the computations of [Ka, Lemma 5.1]. Moreover, we see that $\mathbf{Z}_{N}$ analogue of [Ka, Lemma 5.1] holds in the $\left(\mathscr{A}^{(n)}, \mathbf{Z}_{N}\right)$ case as well. The orbifold weights which we define via the fixed point algebra coincide (via (1.8)) with the $\mathbf{Z}_{2}$ and $\mathbf{Z}_{3}$ examples of [FG], [F], when $N=2,3$, respectively. Also (with $G$-invariant) Boltzmann weights, it is clear that the Yang-Baxter equation in the path algebra

$$
W_{i}(u) W_{i+1}(u+v) W_{i}(v)=W_{i+1}(v) W_{i}(u+v) W_{i+1}(u)
$$

also holds in the orbifold path algebra. In particular, we verify directly the computations of $[\mathrm{FG}, \mathrm{F}]$ that the orbifold weights defined from (1.12) satisfy the YangBaxter equation, and immediately generalise from $N=2,3$ to $N>3$ to get the $\mathscr{A}^{(n)} / \mathbf{Z}_{N}$ models without having to check any identities between theta functions [DZ1, p. 643].

\section{Preliminaries on the Hecke Algebra Subfactors}

Now we recall basic properties of Hecke algebra subfactors of $\mathrm{H}$. Wenzl [We] in the setting of path algebra construction. Section 2 of [DZ1] and Sect. 2 of [So] are good reviews.

Choose the distinguished point $*$ to be the vertex $\Lambda_{1}+\cdots+\Lambda_{N-1}$ of the graph $\mathscr{A}^{(n)}$. We would like to get the following double sequence of the string algebra using a connection:

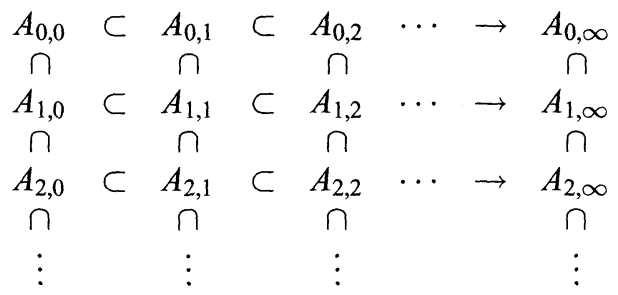

Recall that a connection assigns a complex number to each admissible square.

$$
\begin{array}{lll}
a & \rightarrow & b \\
\downarrow & & \downarrow \\
c & \rightarrow & d
\end{array}
$$

Here the above square is called admissible if $a, b, c, d$ are vertices of the graph and the edges $a \rightarrow b, b \rightarrow d, a \rightarrow c, c \rightarrow d$ come from the graph. A connection produces unitary matrices by which we make identifications for different expressions of strings. See [O3, II.2] for details. 
Connections in paragroups are analogues of Boltzmann weights in solvable lattice model theory without spectral parameters and the unitarity corresponds to the first inversion relations.

For the vertices of the graph of $\mathscr{A}^{(n)}$, we define the inner product by $e_{j} \cdot e_{k}=$ $\delta_{j, k}-\frac{1}{N}$, and set a function $s_{j l}$ by $s_{j l}(\lambda)=\sin \left(\frac{\pi}{n}\left(e_{j}-e_{l}\right) \cdot \lambda\right)$, where $\lambda$ is a vertex of the graph. The connection $W$ is given by

$$
\begin{array}{ccc}
\lambda & \rightarrow & \lambda+e_{k} \\
\downarrow & & \downarrow \\
\lambda+e_{j} & \rightarrow & \lambda+e_{j}+e_{l}
\end{array}=\delta_{j k} \varepsilon+\left(1-\delta_{j l}\right) \bar{\varepsilon} \sqrt{\frac{s_{j l}\left(\lambda+e_{j}\right) s_{j l}\left(\lambda+e_{k}\right)}{s_{j l}(\lambda)^{2}}},
$$

where $\varepsilon=\sqrt{-1} \exp \frac{\pi \sqrt{-1}}{2 n}$ and $k=j$ or $k=l$ is required to get an admissible square. If $j=l$ in this formula, then we have 0 as the denominator of the second term, but we regard the second term to be zero in such a case by the factor $\left(1-\delta_{k l}\right)$. (See [DZ1, (2.16)] and see [O1, O3] for notations on connections.) Using [DZ1, (2.6a)], we can show the unitarity of this connection:

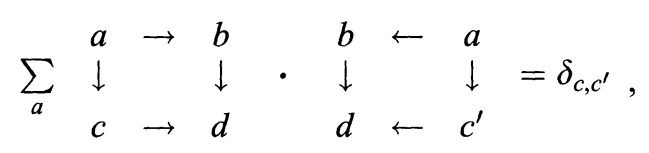

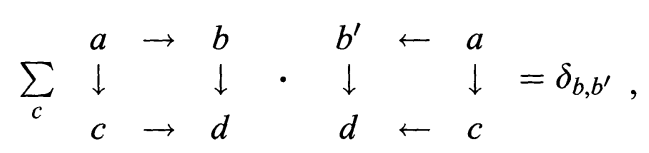

Here and later we use the following conventions:

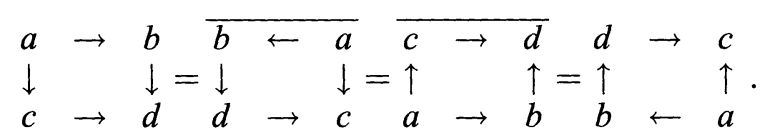
by

$U_{m}^{0}=\sum_{\substack{|\alpha|=m-1 \\ j, k, l}}\left(1-\delta_{j l}\right) \sqrt{\frac{s_{j l}\left(r(\alpha)+e_{j}\right) s_{j l}\left(r(\alpha)+e_{k}\right)}{s_{j l}(r(\alpha))^{2}}}\left(\alpha \cdot e_{j} \cdot e_{l}, \alpha \cdot e_{k} \cdot\left(e_{j}+e_{l}-e_{k}\right)\right)$.

Then these operators $U_{m}^{0}$ satisfy the relations

$$
\begin{aligned}
\left(U_{m}^{0}\right)^{2} & =\beta U_{m}^{0}, \\
U_{m}^{0} U_{m^{\prime}}^{0} & =U_{m^{\prime}}^{0} U_{m}^{0}, \quad \text { if }\left|m-m^{\prime}\right| \geqq 2, \\
U_{m}^{0} U_{m+1}^{0} U_{m}^{0}-U_{m}^{0} & =U_{m+1}^{0} U_{m}^{0} U_{m+1}^{0}-U_{m+1}^{0},
\end{aligned}
$$

where $\beta=2 \cos \frac{\pi}{n}$ as in $[\mathrm{DZ2},(2.6)]$ and the algebra $A_{0, m}$ is generated by $U_{1}^{0}, \ldots, U_{m-1}^{0}$ as in [We]. It will be shown in the proof of Theorem 3.3 that this string algebra double sequence construction gives Wenzl's subfactors in [We]. 
In the case of unoriented graphs, the sequence

$$
A_{0, \infty} \subset A_{1, \infty} \subset A_{2, \infty} \subset A_{3, \infty} \cdots
$$

given by the string algebra double sequence construction is the Jones tower of the subfactor $A_{0, \infty} \subset A_{1, \infty}$ as in [O3, II.6]. But the above double sequence does not produce the Jones tower because our graph is oriented. In order to get the Jones tower, we need the following arguments for "reversing" the orientation of edges.

Denote the entries of the Perron-Frobenius eigenvector of the graph $\mathscr{A}^{(n)}$ by $\mu(\cdot)$ with the normalization $\mu(*)=1$. Then this gives the unique trace on the string algebra as in [E1, O3, II.3] by

$$
\operatorname{tr}(\sigma)=\delta_{\sigma_{+}, \sigma_{-}} \beta^{-|\sigma|} \mu(r(\sigma)), \sigma=\left(\sigma_{+}, \sigma_{-}\right) .
$$

Let

$$
\begin{array}{ll}
a & \rightarrow \\
\downarrow & b \\
c & \rightarrow \\
& \downarrow
\end{array}
$$

be an admissible square. Then we define the values of the following squares:

Definition 2.1.

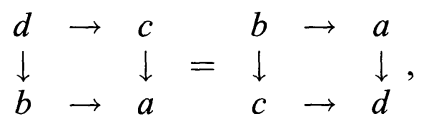

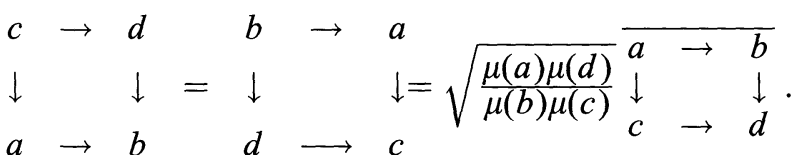

Note that these formulas hold for unoriented graph cases as in [O3, I.3] but now the left-hand sides are undefined because the graph is oriented and the squares on the left-hand sides are not admissible. We need unitarity also for these newly defined connections.

Proposition 2.2. For connections in Definition 2.1, we have unitarity identities (2.2) with conventions (2.3).

Proof. First we have to prove the following:

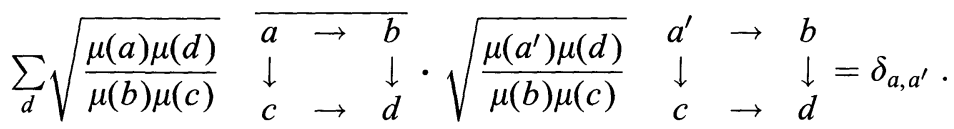

Note that the diagram

$$
\begin{array}{ccc}
A_{0, m} & \subset & A_{0, m+1} \\
\cap & & \cap \\
A_{1, m} & \subset & A_{1, m+1}
\end{array}
$$

gives a commuting square in the sense of [GHJ, Sect. 4.2] by [We, Proposition 3.2]. Then Ocneanu's computation at the end of [O2] produces the above equality. (This can be easily checked directly with formulas for the conditional expectations in [O3, II.3]. Also see [HS, Sc, 1.1].) 
If we fix $b$ and $c$ in the above formula, the numbers of possible $a$ and $d$ are equal. This implies the other identities. (That is, an isometric square matrix is a unitary.)

Q.E.D.

Ocneanu's computation cited above means that in the string algebra situations, the commuting square condition corresponds to the second inversion relations in solvable lattice models. (See [Ba] for the inversion relations.)

With these new definitions, we construct a double sequence of string algebras (1.1) as follows. For the inclusions

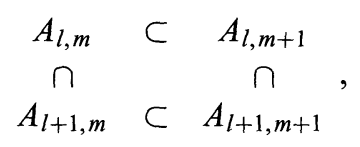

we use the graph $\mathscr{A}^{(n)}$ and the original connection if $l$ is even, and use the graph $\mathscr{A}^{(n)}$ for the horizontal edges and the graph $\tilde{\mathscr{A}}^{(n)}$ with the orientation reversed for the vertical edges and the new connection of $l$ is odd. That is, if $l$ is odd, we have the following type of squares:

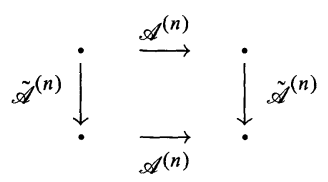

The vertices of $\mathscr{A}^{(n)}$ can be colored by $N$ colors in $\mathbf{Z} / N \mathbf{Z}=\{0,1, \ldots N-1\}$ so that the starting vertex $*$ has color 0 and each oriented edge goes from a color $k$ to a color $k+1, k \in \mathbf{Z} / N \mathbf{Z}$. Take a subgraph $\mathscr{A}_{k}^{(n)}$ of $\mathscr{A}^{(n)}$ which has vertices of colors $k$ and $k+1$ and edges connecting these vertices. We regard $\mathscr{A}_{k}^{(n)}$ as an unoriented graph. In this way, the above setting can be described as follows: For horizontal edges, we always use the original (oriented) graph $\mathscr{A}^{(n)}$ and for the vertical edges we use the (unoriented) graphs $\mathscr{A}_{m}^{(n)}$. Thus in the diagram (1.1), the colors of vertices corresponding to each algebra are illustrated as follows:

$$
\begin{array}{cccccccc}
0 & 1 & 2 & \cdots & N-1 & 0 & 1 & \cdots \\
1 & 2 & 3 & \cdots & 0 & 1 & 2 & \cdots \\
0 & 1 & 2 & \cdots & N-1 & 0 & 1 & \cdots \\
1 & 2 & 3 & \cdots & 0 & 1 & 2 & \cdots \\
0 & 1 & 2 & \cdots & N-1 & 0 & 1 & \cdots \\
\vdots & \vdots & \vdots & \cdots & \vdots & \vdots & \vdots & \cdots
\end{array}
$$

Note that the Perron-Frobenius eigenvector $\mu$ also gives a Perron-Frobenius eigenvector for each $\mathscr{A}_{k}^{(n)}$ because the incidence matrix of the graph $\mathscr{A}^{(n)}$ is normal. (See the following example.) 


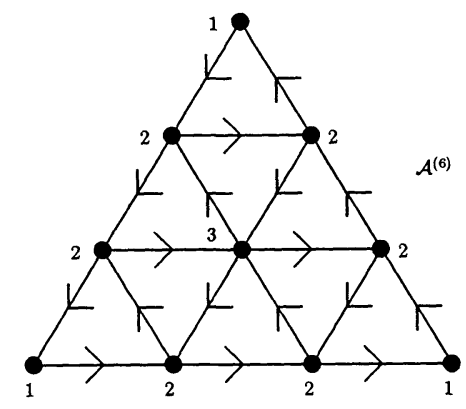

$\Downarrow$

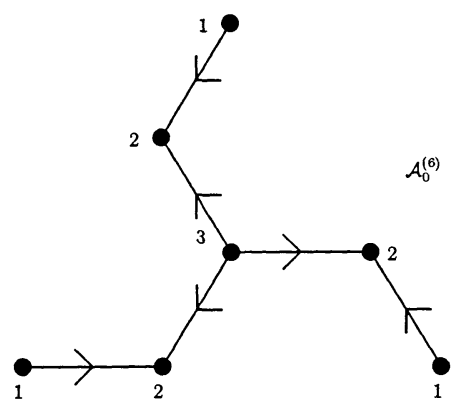

Fig. 2.1

Because the vertical graphs $\mathscr{A}_{k}^{(n)}$ are unoriented, we can define the Jones projections in the vertical string algebras by the same formula as in [O3, II.3]:

$$
e_{l}=\sum_{\substack{|\alpha|=l-1 \\|v|=|w|=1}} \frac{1}{\beta} \frac{\mu(r(v))^{1 / 2} \mu(r(w))^{1 / 2}}{\mu(r(\alpha))}(\alpha \cdot v \cdot \tilde{v}, \alpha \cdot w \cdot \tilde{w}),
$$

where $\beta$ is the Perron-Frobenius eigenvalue for the vertical graph. As in [O3, II.5] we get flatness of these vertical Jones projections by Definition 2.1. That is, if we embed $e_{l} \in A_{l+1,0}$ into $A_{l+1, m}$ by putting trivial tails and transform it to the form horizontal edges of length $m$ followed by vertical edges of length $l+1$ using the connection, we get the following expression:

$$
e_{l}=\sum_{\substack{|\alpha|=m \\\left|\alpha^{\prime}\right|=l-1 \\|v|=|w|=1}} \frac{1}{\beta} \frac{\mu(r(v))^{1 / 2} \mu(r(w))^{1 / 2}}{\mu(r(\alpha))}\left(\alpha \cdot \alpha^{\prime} \cdot v \cdot \tilde{v}, \alpha \cdot \alpha^{\prime} \cdot w \cdot \tilde{w}\right),
$$

where $\alpha$ is horizontal and $\alpha^{\prime}$ is vertical.

Denote the conditional expectation from $A_{l+1, \infty}$ onto $A_{l, \infty}$ by $E_{l}$. Note that if $x \in A_{l+1, m}$, then $E_{l}(x)$ is given by the conditional expectation of $x$ onto $A_{l, m}$ because of the commuting square condition. Then it is easy to see that the above Jones projections satisfy 
(1) $e_{l} x=x e_{l}$, for $x \in A_{l-1, \infty}$.

(2) $e_{l} x e_{l}=E_{l-1}(x) e_{l}$, for $x \in A_{l, \infty}$.

(3) $<A_{l-1, \infty}, e_{l}>=A_{l, \infty}$.

Thus if we set $N=A_{0, \infty}$ and $M=A_{1, \infty}$ then the sequence

$$
A_{0, \infty} \subset A_{1, \infty} \subset A_{2, \infty} \subset A_{3, \infty} \subset A_{4, \infty} \subset \cdots
$$

can be identified with the Jones tower

$$
N \subset M \subset M_{1} \subset M_{2} \subset M_{3} \subset \cdots,
$$

by [PP, Proposition 1.2].

At the end of this section, we prove certain technical properties of the original connections on the graph $\mathscr{A}^{(n)}$. These for the case $N=2$ correspond to Lemmas $4.3,4.4,4.5$ in [Ka].

Lemma 2.3. For the original connection $W$ on $\mathscr{A}^{(n)}$, we have the following identities:

$$
\begin{aligned}
& \downarrow \quad\rfloor^{\lambda+e_{j}}=(-1)^{\left(e_{j}-e_{k}\right) \cdot \lambda} \frac{\sin \frac{\pi}{n}}{\sin \frac{\left(\left(e_{j}-e_{k}\right) \cdot \lambda\right) \pi}{n}} \varepsilon^{-2\left(e_{j}-e_{k}\right) \cdot \lambda-1}, \\
& \lambda+e_{j} \longrightarrow \lambda+e_{j}+e_{k} \\
& \lambda \quad \longrightarrow \quad \lambda+e_{k} \\
& \downarrow \downarrow 1=\sqrt{\frac{s_{j k}\left(\lambda+e_{j}\right) s_{j k}\left(\lambda+e_{k}\right)}{s_{j k}(\lambda)^{2}}} \bar{\varepsilon}, \\
& \lambda+e_{j} \longrightarrow \lambda+e_{j}+e_{k}
\end{aligned}
$$

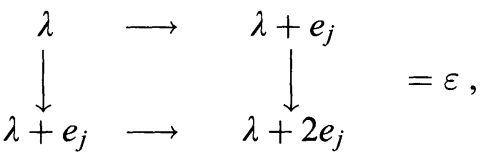

where we assume $j \neq k$ in the first and the second cases.

Proof. These are verified directly using the definition of $W$ and the sine law. Note that $\left(\varepsilon_{j}-e_{k}\right) \cdot \lambda$ is a non-zero integer for $j \neq k$.

Q.E.D

Next we consider a large diagram. As in [O3, II.3] or [Ka, Sect. 1], a large diagram means the sum of the products of connection values over all the configurations. (See Sect. 1 for its similarity to partition functions. We also call them partition functions.) The arguments of partition functions are determined up to $\pi$ as follows.

Proposition 2.4. The value of an $l \times k$-diagram

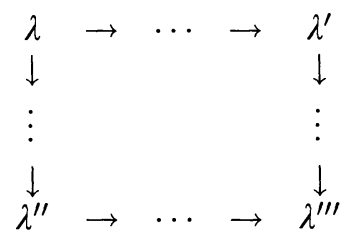


is in the set

$$
\mathbf{R} \cdot \varepsilon^{\lambda \cdot \lambda+\lambda^{\prime \prime \prime} \cdot \lambda^{\prime \prime \prime}-\lambda^{\prime} \cdot \lambda^{\prime}-\lambda^{\prime \prime} \cdot \lambda^{\prime \prime}-k l(N-2) / N} .
$$

Proof. If $k=l=1$, this follows directly from Lemma 2.3. The general case can be proved by induction.

Q.E.D.

\section{The Yang-Baxter Equation and Flatness}

In this section, we compute the canonical commuting squares (in the sense of Popa [P3]) for subfactors of Wenzl [We] using solutions to the Yang-Baxter equations by Jimbo-Miwa-Okado [JMO1, JMO2]. We also show some computations of partition functions follow from the Yang-Baxter equation - indeed vertical parallel transport for Wenzl subfactors and their orbifolds is closely related to the Yang-Baxter equation (Lemma 3.1).

Some parts of this section seem to be known to several specialists. (Wenzl mentioned the use of the $R$-matrix version of the Yang-Baxter equation for computations of higher relative commutants at the end of his paper [We] and the second author saw Wenzl show some examples of the principal graphs of his subfactors in his seminar talk, but he was unable to compute the canonical commuting squares.) However, we have been unable to find actual computations of these in the literature, and we will need several explicit formulae later in this paper, so we will present the details here.

First we show that the connection $W$ satisfies the Yang-Baxter equation, or the star-triangle relation, without a spectral parameter. This means the following. Take a hexagon

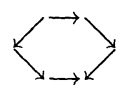

where the vertices and edges are taken from the graph $\mathscr{A}^{(n)}$. For each such a hexagon, we first consider configurations tiply these three connection values of the parallelograms and make a sum of these products over all the configurations. Similarly we make another sum over configurations with the same boundary conditions. Then our claim is that these two numbers coincide. We state this as a proposition and prove it.

Proposition 3.1. The connection $W$ on $\mathscr{A}^{(n)}$ satisfies the Yang-Baxter equation in the above sense.

Proof. We use solutions of Jimbo-Miwa-Okado. Let $p \rightarrow 0$ and $L=n$ in their formulas [JMO2, $(2.4 \mathrm{a}-\mathrm{c})]$. Then the solutions are now Laurent polynomials of $x=$ $\exp \left(\frac{\pi u \sqrt{-1}}{n}\right)$ and the highest terms have degree 1 . Define another connection $W^{\prime}$ by taking a coefficient of $x$ and multiplying it by $-2 \sqrt{-1} \bar{\varepsilon} \sin \frac{\pi}{n}$. Direct computations show the following identities: 


$$
\begin{aligned}
& W^{\prime}\left(\begin{array}{ccc}
\lambda & \longrightarrow & \lambda+e_{j} \\
\downarrow & & \downarrow \\
\lambda+e_{j} & \longrightarrow & \lambda+e_{j}+e_{k}
\end{array}\right)=(-1)^{\left(e_{j}-e_{k}\right) \cdot \lambda} \frac{\sin \frac{\pi}{n}}{\sin \frac{\left(\left(e_{j}-e_{k}\right) \cdot \lambda\right) \pi}{n}} \varepsilon^{-2\left(e_{j}-e_{k}\right) \cdot \lambda-1}, \\
& W^{\prime}\left(\begin{array}{ccc}
\lambda & \longrightarrow & \lambda+e_{k} \\
\downarrow & & \downarrow \\
\lambda+e_{j} & \longrightarrow & \lambda+e_{j}+e_{k}
\end{array}\right)=\sqrt{\frac{s_{j k}\left(\lambda+e_{j}\right) s_{j k}\left(\lambda+e_{k}\right)}{s_{j k}(\lambda)^{2}}} \bar{\varepsilon}, \\
& W^{\prime}\left(\begin{array}{ccc}
\lambda & \longrightarrow & \lambda+e_{j} \\
\downarrow & & \\
\lambda+e_{j} & \longrightarrow & \lambda+e_{j}+e_{j}
\end{array}\right)=\varepsilon,
\end{aligned}
$$

where $j \neq k$ in the first and the second cases. Comparing these and formulas in Lemma 2.3, we know that $W$ and $W^{\prime}$ coincide in the first and the third cases and differ only by sign in the second case. The above definition of $W^{\prime}$ shows that the Yang-Baxter equation holds for $W^{\prime}$. Looking at the sign difference between $W$ and $W^{\prime}$ carefully for all the possible cases, we can conclude that the Yang-Baxter equation also holds for $W$.

We thank Professor M. Okado for explaining the above method to us. See [M, Sect. 1] for a similar computation.

Next we define another connection $W^{\prime \prime}$ by

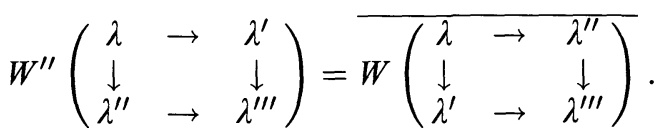

Lemma 3.2. The connection $W^{\prime \prime}$ and the new connection defined in Definition 2.1 satisfy the star-triangle relation.

Proof. Note that each of two ways of configurations, we take downward edges from the oriented graph $\mathscr{A}^{(n)}$ and horizontal edges from the $\tilde{\mathscr{A}}^{(n)}$ with the orientation reversed. Thus in the configurations $W^{\prime}$ and the right two parallelograms have values defined in Definition 2.1, and in the other configurations left two parallelograms have values defined in Definition 2.1.

Then we get the desired equalities by taking complex conjugates of the original star-triangle relation in Proposition 3.1 and multiplying the both hand sides by certain terms involving $\mu(\cdot)$.

Q.E.D.

We can prove the following characterization of the tower of the relative commutants.

Theorem 3.3. In the string algebra double sequence $\left(A_{k, l}\right)$ discussed at the end of Sect. 2, we have $A_{0, \infty}^{\prime} \cap A_{k, \infty}=A_{k, 0}$.

Proof. First we prove $A_{0, \infty}^{\prime} \cap A_{k, \infty} \supset A_{k, 0}$.

We set $\sigma_{m}^{0}=\varepsilon+\bar{\varepsilon} \beta U_{m}^{0}$ for $U_{m}^{0}$ in Sect. 2. Because these generate $A_{0, \infty}$, it is enough to prove that each $\sigma_{m}^{0}$ commutes with $A_{k, 0}$. The operator $\sigma_{m}^{0}$ is a unitary because $\beta=-\varepsilon^{2}-\bar{\varepsilon}^{2}$. (Note that our $\varepsilon^{4}$ and $\sigma_{m}^{0}$ correspond to $q$ and $\varepsilon^{-3} g_{m}$ in 
[We, Sect. 2].) Because the formula for $\sigma_{m}^{0}$ has the same form as the definition of the connection $W$, this is a face operator in the sense of [R, page 400]. We also define $\sigma_{m}^{1}$ similarly for the string algebras $A_{0,0} \subset A_{1,0} \subset A_{1,1} \subset A_{1,2} \cdots$.

Because we have the star-triangle relation, the identification using the connection transforms $\sigma_{m}^{0}$ to $\sigma_{m+1}^{1}$, which is also a face operator, by [R, Proposition 5]. This means that $\sigma_{m}^{0}$ commutes with $A_{1,0}$.

Similarly, by the other star-triangle relation in Lemma 3.2, we get that $\sigma_{m+1}^{1}$ is identified with a face operator in the next step, that is, a face operator for the second row

$$
A_{2,0} \subset A_{2,1} \subset A_{2,2} \subset A_{2,3} \cdots
$$

of the double sequence. This means that $\sigma_{m}^{0}$ commutes with $A_{2,0}$.

Repeating these two cases inductively, we get $A_{0, \infty}^{\prime} \cap A_{k, \infty} \subset A_{k, 0}$. 1.6].

For the other inclusion, we apply Wenzl's dimension estimate [We, Theorem

Q.E.D.

The above proof means that the subfactor $A_{0, \infty} \subset A_{1, \infty}$ is expressed as $\left\langle\sigma_{2}^{1}, \sigma_{3}^{1}\right.$, $\left.\sigma_{4}^{1}, \ldots\right\rangle \subset\left\langle\sigma_{1}^{1}, \sigma_{2}^{1}, \sigma_{3}^{1}, \ldots\right\rangle$. Because the above representations of the Hecke algebra are the same as the $(N, n)$ - representations in [We, Sect. 2], this string algebra construction gives Wenzl's subfactors in [We, Theorem 3.7] with the index $\frac{\sin ^{2} \frac{N \pi}{n}}{\sin ^{2} \frac{\pi}{n}}$.

As a direct corollary to the above, we get the following:

Corollary 3.4. For the above subfactors of Wenzl, the principal graph is given by $\mathscr{A}_{0}^{(n)}$.

Proof. This is clear because the vertical string algebra sequence

$$
A_{0,0} \subset A_{1,0} \subset A_{2,0} \subset A_{3,0} \cdots
$$

is given by the unoriented graph $\mathscr{A}_{0}^{(n)}$.

Here are some examples of the principal graphs. (The second author saw H. Wenzl show these graphs without proofs in his talk at the Mittag-Leffler Institute in September, 1988.)
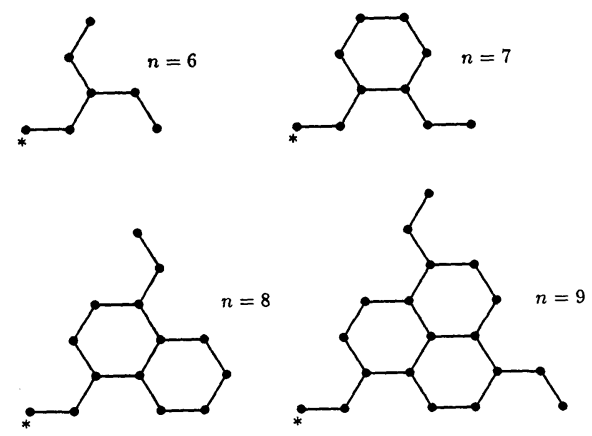

Fig. 3.1

M. Izumi [I, Figs. 5, 7] computed the principal graphs for subfactors constructed in [We, page 360] with index $\frac{\sin ^{2} \frac{n \pi}{N}}{\sin ^{2} \frac{\pi}{N}}$ for the case $n=3$. (These are constructed by 
cutting the subfactor $N \subset M_{1}$ by a minimal projection in $N^{\prime} \cap M_{1}$ for the subfactor $N \subset M$ of type $A_{N-1}$. These are also the same as subfactors in [GHJ, Sect. 4.5] for diagrams $A_{n}$. So Okamoto's method [Ok] also gives the principal graphs.) Figure 3.1 shows that the above subfactors are different from these because they have the different principal graphs while the indices are equal.

With further work, we can compute the canonical commuting squares for these subfactors as follows.

In the above construction, we used the graphs $\mathscr{A}^{(n)}, \tilde{\mathscr{A}}^{(n)}, \mathscr{A}^{(n)}, \tilde{\mathscr{A}}^{(n)}$, and so on, in this order, for the vertical directions. Next we construct another double sequence of string algebras starting from $*$ by using the graphs $\tilde{\mathscr{A}}^{(n)}, \mathscr{A}^{(n)}, \tilde{\mathscr{A}}^{(n)}, \mathscr{A}^{(n)}$, and so on, in this order, for the vertical direction. We label these as follows:

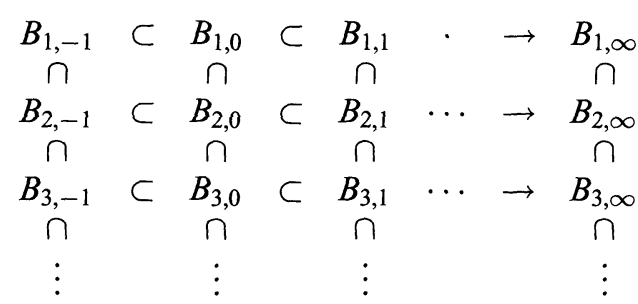

For each of the inclusions $B_{1,-1} \subset B_{1,0}$ and $A_{0,0} \subset A_{1,0}$, we have a single edge. By identifying these, we can identify $A_{k, l}$ and $B_{k, l}$ for $k \geqq 1, l \geqq 0$. In this way, we get the following double sequence.

$$
\begin{array}{ccccccccccc} 
& & A_{0,0} & \subset & A_{0,1} & \subset & A_{0,2} & \cdots & \rightarrow & N \\
& & \cap & & \cap & & \cap & & & \cap \\
A_{1,-1} & \subset & A_{1,0} & \subset & A_{1,1} & \subset & A_{1,2} & \cdots & \rightarrow & \rightarrow \\
\cap & & \cap & & \cap & & \cap & & & \cap \\
A_{2,-1} & \subset & A_{2,0} & \subset & A_{2,1} & \subset & A_{2,2} & \cdots & \rightarrow & M_{1} \\
\cap & & \cap & & \cap & & \cap & & & \cap \\
\vdots & & \vdots & & \vdots & & \vdots & & & & \vdots
\end{array}
$$

Note that in the above diagram, the colors of vertices corresponding to each algebra are illustrated as follows:

$$
\begin{array}{ccccccccc} 
& 0 & 1 & 2 & \cdots & N-1 & 0 & 1 & \cdots \\
0 & 1 & 2 & 3 & \cdots & 0 & 1 & 2 & \cdots \\
N-1 & 0 & 1 & 2 & \cdots & N-1 & 0 & 1 & \cdots \\
0 & 1 & 2 & 3 & \cdots & 0 & 1 & 2 & \cdots \\
N-1 & 0 & 1 & 2 & \cdots & N-1 & 0 & 1 & \cdots \\
\vdots & \vdots & \vdots & \vdots & \cdots & \vdots & \vdots & \vdots & \cdots
\end{array}
$$

The same arguments as in the proof of Theorem 3.3 shows $M_{k} \cap M^{\prime}=A_{k+1,-1}$. Thus we can determine the canonical commuting squares in the sense of [P, Sect. 6] for the above subfactors as follows.

Theorem 3.5. For the above subfactor $N \subset M$, the canonical commuting squares are given by

$$
\begin{array}{ccccccc}
A_{1,-1} & \subset & A_{2,-1} & \subset & A_{3,-1} & \subset & \cdots \\
A_{1,0} & \subset & A_{2,0} & \subset & A_{3,0} & \subset & \cdots
\end{array}
$$


This means that only the subgraph connecting vertices with colors $N-1,0,1$ is essential and the other parts of the graph are redundant from the operator algebraic viewpoint. We also have the following corollary.

Corollary 3.6. For the above subfactors, the "dual" principal graph is given by $\mathscr{A}_{N-1}^{(n)}$, which is isomorphic to $\mathscr{A}_{0}^{(n)}$.

Note that $\mathscr{A}_{0}^{(n)}$ and $\mathscr{A}_{N-1}^{(n)}$ are isomorphic as graphs, but the way these are connected is non-trivial. (Consider the Bratteli diagrams for the sequence in Theorem 3.5.) This means that "contragredient" map in the sense of Ocneanu [O1, page 150] is non-trivial. See the following example.

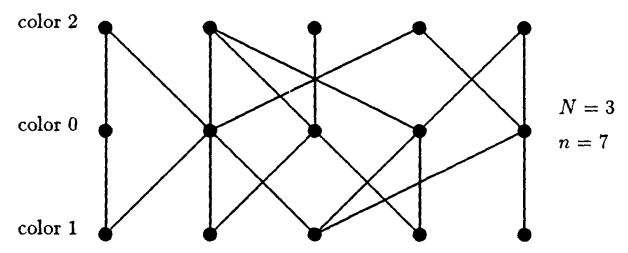

Fig. 3.2

Note that a trivial contragredient map would produce the following graph.

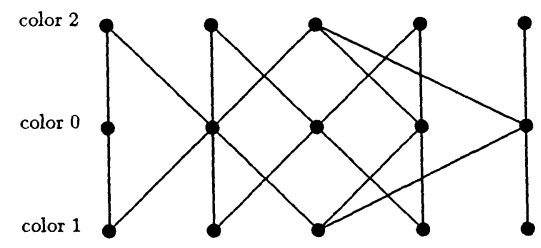

Fig. 3.3

\section{Orbifold Construction}

We set $A_{0}=*$ and label the other end vertices of the graph $\mathscr{A}^{(n)}$ by $A_{1}=$ $A_{0}+(n-N) e_{1}, A_{2}=A_{1}+(n-N) e_{2}, \ldots, A_{N-1}=A_{N-2}+(n-N) e_{N-1}$.

Define a rotation symmetry $\rho$ of the graph $\mathscr{A}^{(n)}$ by

$$
\rho\left(A_{j}+\sum_{k} c_{k} e_{k}\right)=A_{j+1}+\sum_{k} c_{k} e_{k+1},
$$

where indices are in $\mathbf{Z} / N \mathbf{Z}$ and $c_{k} \in \mathbf{C}$. Note that $\rho^{N}=i d$ and the connection $W$ is invariant under this $\rho$ :

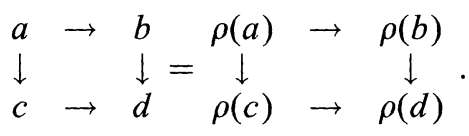

Next we assume $n \equiv 0(\bmod N)$, and construct a double sequence of the string algebra as in Sect. 2, but we allow any of $A_{j}$ as starting points of edges. That is, we took a string $\left(\rho_{+}, \rho_{-}\right)$with $s\left(\rho_{+}\right)=s\left(\rho_{-}\right)=*, r\left(\rho_{+}\right)=r\left(\rho_{-}\right),\left|\rho_{+}\right|=\left|\rho_{-}\right|$in 
Sect. 2, but now we drop the requirement $s\left(\rho_{+}\right)=s\left(\rho_{-}\right)=*$ and instead impose $s\left(\rho_{+}\right), s\left(\rho_{-}\right) \in\left\{A_{0}, \ldots, A_{N-1}\right\}$. The multiplication is defined by the same formula as usual. Note that we have a connected Bratteli diagram by the assumption $n \equiv 0$ $(\bmod N)$. We label this double sequence as follows:

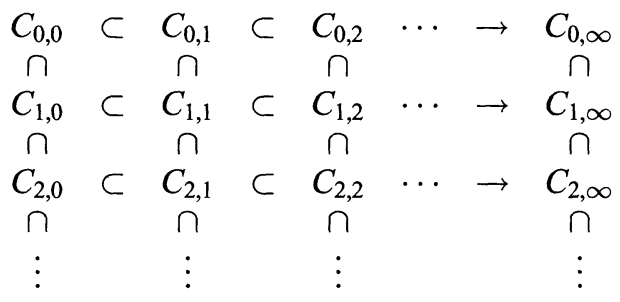

Note that $C_{0,0}$ has $N$ copies of $\mathbf{C}$ and that as in Sect. 2, for the inclusions

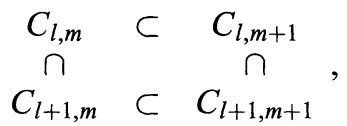

we use the graph $\mathscr{A}^{(n)}$ and the original connection if $l$ is even, and use the graph $\mathscr{A}^{(n)}$ for the horizontal edges and the graph $\tilde{\mathscr{A}}^{(n)}$ with the orientation reversed for the vertical edges and the new connection of $l$ is odd. (The case $N=2$ of this construction appeared in [Ka, Sect. 5], in which case, the original graph is a Dynkin diagram of type $A$.)

We can apply $\rho$ to each $C_{l, m}$ as a $*$-algebra isomorphism and denote it by $\rho$ again. (Because the connection is invariant under $\rho$, the identifications based on the connection are compatible with this $\rho$, thus this automorphism is well-defined.) Set $D_{l, m}=C_{l, m}^{\rho}$, the fixed point algebra under $\rho$. Set $D_{l, \infty}$ to be the weak closure of $\bigcup_{m} D_{l, m}$ in its GNS-representation with respect to the trace and we study double sequence of $D_{l, m}$. Because the vertical Jones projections $e_{l}$ for the double sequence $\left(C_{l, m}\right)$ are invariant under $\rho$ by definition, we get $e_{l} \in D_{l, \infty}$. An argument as in Sect. 2 shows that $D_{l, \infty}=\left\langle D_{l-1, \infty}, e_{l-1}\right\rangle$ and

$$
D_{0, \infty} \subset D_{1, \infty} \subset D_{2, \infty} \subset D_{3, \infty} \subset \cdots
$$

is the Jones tower for the subfactor $D_{0, \infty} \subset D_{1, \infty}$, and

$$
\left[D_{1, \infty}, D_{0, \infty}\right]=\left[C_{1, \infty}, C_{0, \infty}\right]=\left[A_{1, \infty}, A_{0, \infty}\right]=\frac{\sin ^{2} \frac{N \pi}{n}}{\sin ^{2} \frac{\pi}{n}}
$$

(This can be regarded as a very special case of the Invariance Principle of A.J. Wassermann [Wa, page 227] or [GHJ, Lemma 4.7.1].)

Our construction of $C_{l m}$ may look artificial, but we prove that the subfactor $C_{0, \infty} \subset C_{1, \infty}$ is conjugate to the original construction.

Proposition 4.1. The subfactor $C_{0, \infty} \subset C_{1, \infty}$ is conjugate to the original Hecke algebra subfactor of Wenzl, and hence the subfactor $D_{0, \infty} \subset D_{1, \infty}$ is realized as simultaneous fixed point algebras of the Hecke algebra subfactors of Wenzl by the $\mathbf{Z}_{N}$-action. 
Proof. It is clear that the original Hecke algebra subfactor is obtained by cutting $C_{0, \infty} \subset C_{1, \infty}$ by the projection in $C_{0,0}$ corresponding to $*$. In $C_{0, \infty}$, the sequence of the face operators $\left\{W_{n}\right\}$ is central for $C_{1, \infty}$. If we shift this sequence by 1 , we get another central sequence which does not commute with the original central sequence. So by [Bi], our subfactor $C_{0, \infty} \subset C_{1, \infty}$ has simultaneous splittings of a common hyperfinite $\mathrm{II}_{1}$ factor, which is called the relative McDuff condition. This implies the conclusion. (Also see [P2, page 200].)

Q.E.D.

We call these subfactors $D_{0, \infty} \subset D_{1, \infty}$ orbifold subfactors. (This construction is related to orbifold models in solvable lattice model theory [DZ1, DZ2, F, FG, Ko, $\mathrm{Z}$ ] and M. Choda's work [C] on duality of graphs.) We would like to compute the higher relative commutants for this subfactor. By $n \equiv 0(\bmod N)$, there is a unique vertex $C$ in $\mathscr{A}^{(n)}$ that is invariant under $\rho$ and all the vertices $A_{j}$ 's have color 0 . Note that the center $C$ is expressed as

$$
A_{0}+\frac{n-N}{N}\left((N-1) e_{1}+(N-2) e_{2}+\cdots+e_{N-1}\right)
$$

(See the following example.)

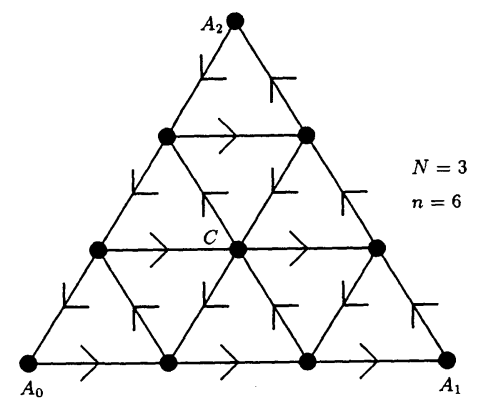

Fig. 4.1

We would like to show the vertical string algebra sequence

$$
D_{0,0} \subset D_{1,0} \subset D_{2,0} \subset D_{3,0} \cdots
$$

gives the higher relative commutants as in Theorem 3.3 under some appropriate conditions. For this, it is enough to prove $\sigma \sigma^{\prime}=\sigma^{\prime} \sigma$ for $\sigma \in D_{0, m}, \sigma^{\prime} \in D_{l, 0}$, because we can apply Wenzl's estimate [We, Theorem 1.6] again to get the reversed inclusion.

We work in more details on the equality $\sigma \sigma^{\prime}=\sigma^{\prime} \sigma$. Let $\alpha, \beta$ be paths with the same length on $\mathscr{A}^{(n)}$ and with $s(\alpha)=A_{0}, s(\beta)=A_{j}, r(\alpha)=r(\beta)=B_{0}$, where $B_{0}$ is some vertex of $\mathscr{A}^{(n)}$. Set $\rho^{l}\left(B_{0}\right)=B_{l}$ and $\sigma=\sum_{l=0}^{N-1}\left(\rho^{l}(\alpha), \rho^{l}(\beta)\right)$. (See the following example.) 


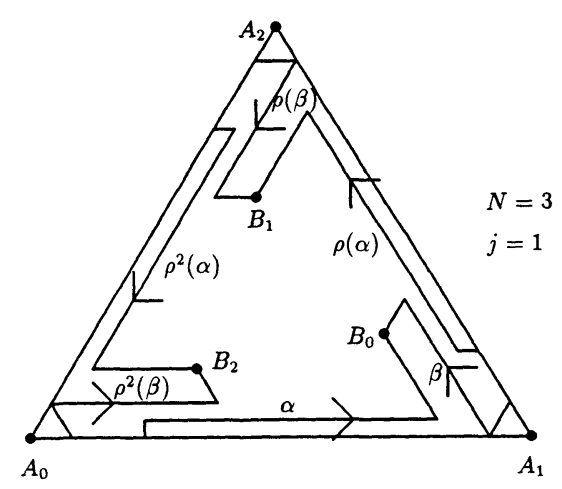

Fig. 4.2

Note that $\sigma$ 's of the above form span the $\bigcup_{m} D_{0, m}$. Similarly take paths $\alpha^{\prime}, \beta^{\prime}$ with the same length on the graph $\mathscr{A}_{0}^{(n)}$ without orientation and with $s(\alpha)=A_{0}, s(\beta)=A_{k}, r(\alpha)=r(\beta)=C_{0}$, where $C_{0}$ is some vertex of $\mathscr{A}_{0}^{(n)}$. Setting $\rho^{l}\left(C_{0}\right)=C_{l}$ and $\sigma^{\prime}=\sum_{l=0}^{N-1}\left(\rho^{l}\left(\alpha^{\prime}\right), \rho^{l}\left(\beta^{\prime}\right)\right)$, we again have that the vertical string algebra $\bigcup_{m} D_{m, 0}$ is spanned by $\sigma^{\prime}$ 's of the above form.

Thus we have to study under what conditions we get $\sigma \sigma^{\prime}=\sigma^{\prime} \sigma$. We need a lemma here.

Lemma 4.2. We have the identity

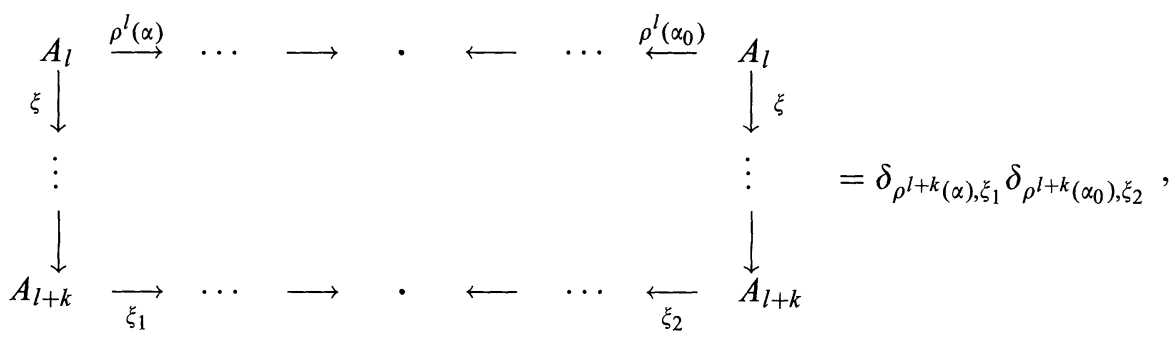

for paths $\alpha, \alpha_{0}$ on $\mathscr{A}^{(n)}$ and $\zeta$ on $\mathscr{A}_{0}^{(n)}$.

Proof. Without loss of generality, we may assume that $l=0$. The equality means that the string $\left(\alpha, \alpha_{0}\right)$ moves to $\rho^{k}\left(\alpha, \alpha_{0}\right)$ under the vertical parallel transport from $A_{0}$ to $A_{k}$. This is so because the face operators move to face operators as in the proof of Theorem 3.3, the face operators generate the entire string algebra, and the face operators are invariant under $\rho$.

Q.E.D.

By embedding $\sigma$ and $\sigma^{\prime}$ into the same algebra and using Lemma 4.2, we get the following two expressions: 
Orbifold Subfactors from Hecke Algebras

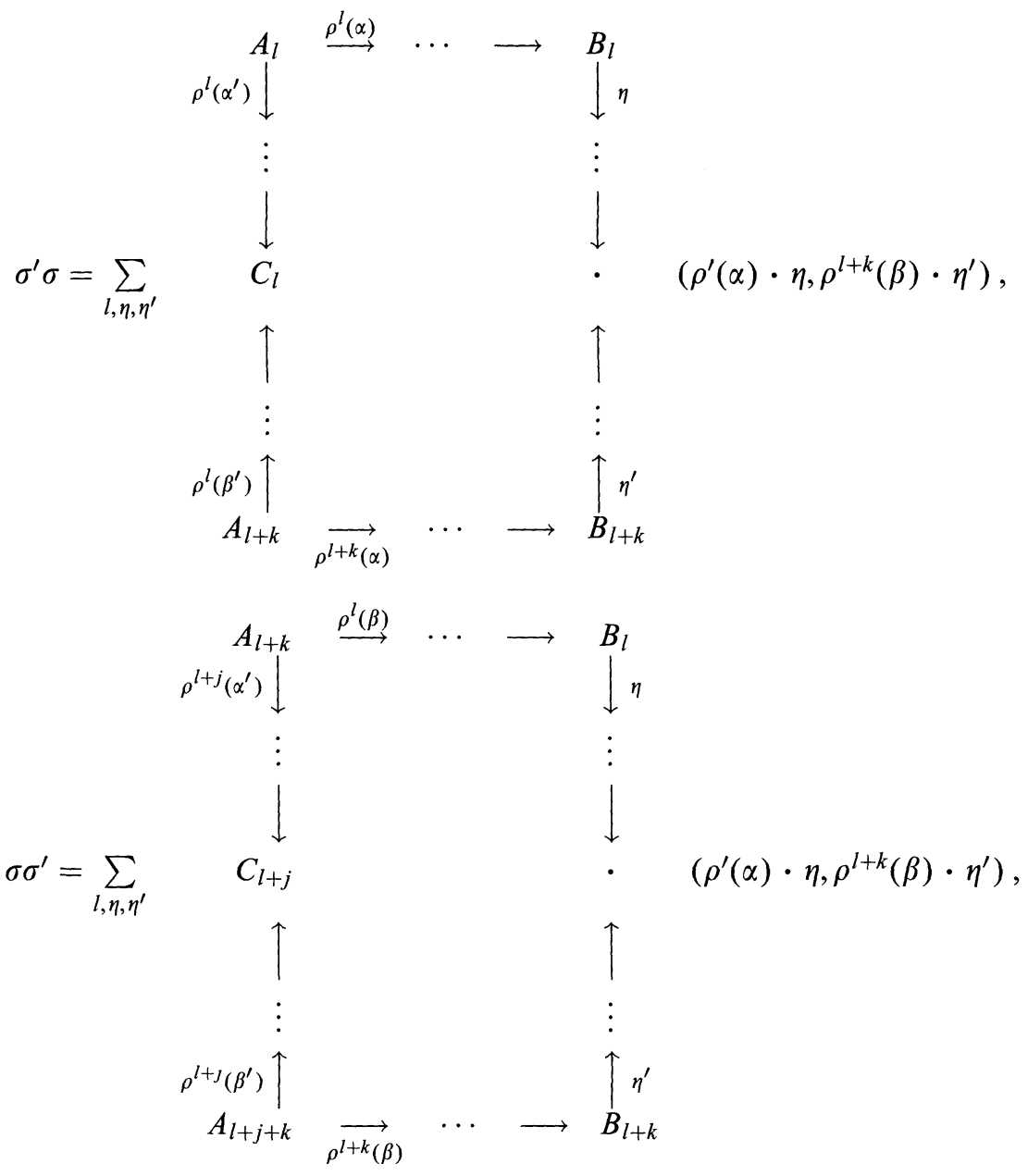

where $\eta, \eta^{\prime}$ are vertical strings with $r(\eta)=r\left(\eta^{\prime}\right)$. These imply that the identity $\sigma \sigma^{\prime}=\sigma^{\prime} \sigma$ is equivalent to the following identities.

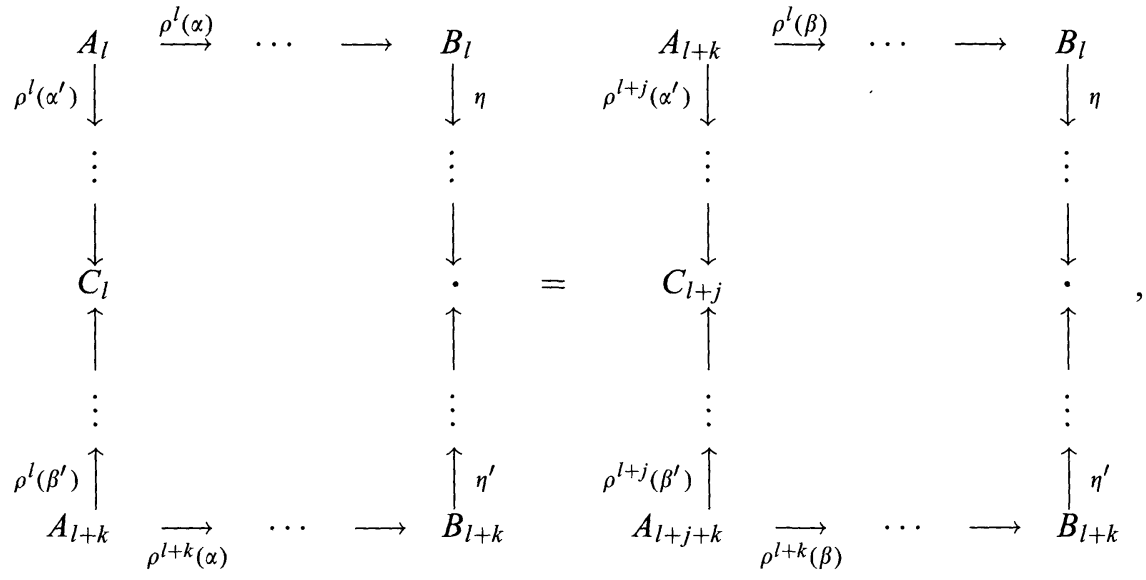

for all $l, \eta, \eta^{\prime}$. 
We change the orientations of vertical arrows of the lower halves of the diagrams, take the squares of the difference of the both hand sides of (4.1), and then take a sum over all $\eta, \eta^{\prime}$. The equality $\sigma \sigma^{\prime}=\sigma^{\prime} \sigma$ is equivalent to this sum being 0 . By expanding the summation, we get the following identity. (See [Ka, Lemma 5.2] for this method in the case $N=2$.)

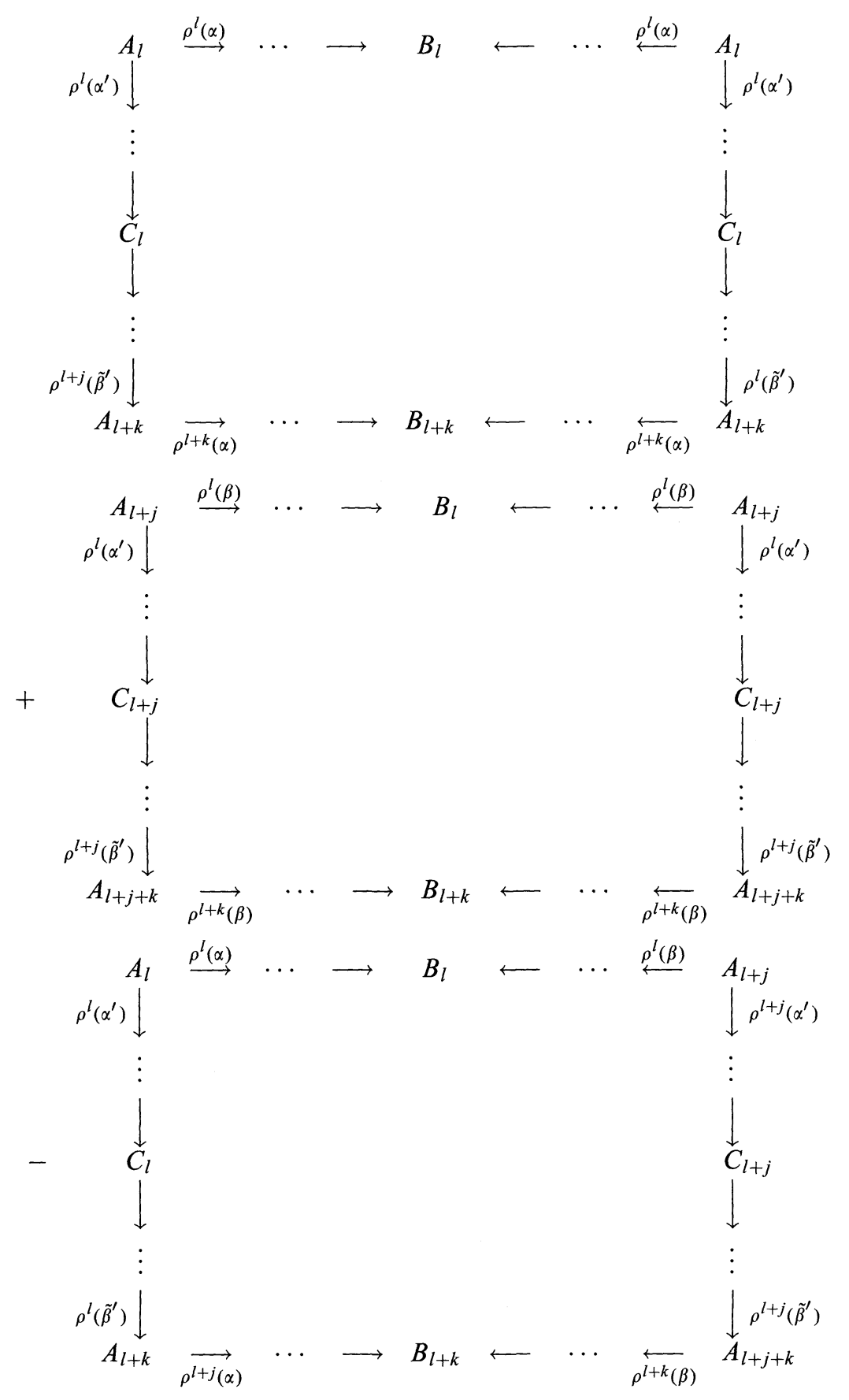




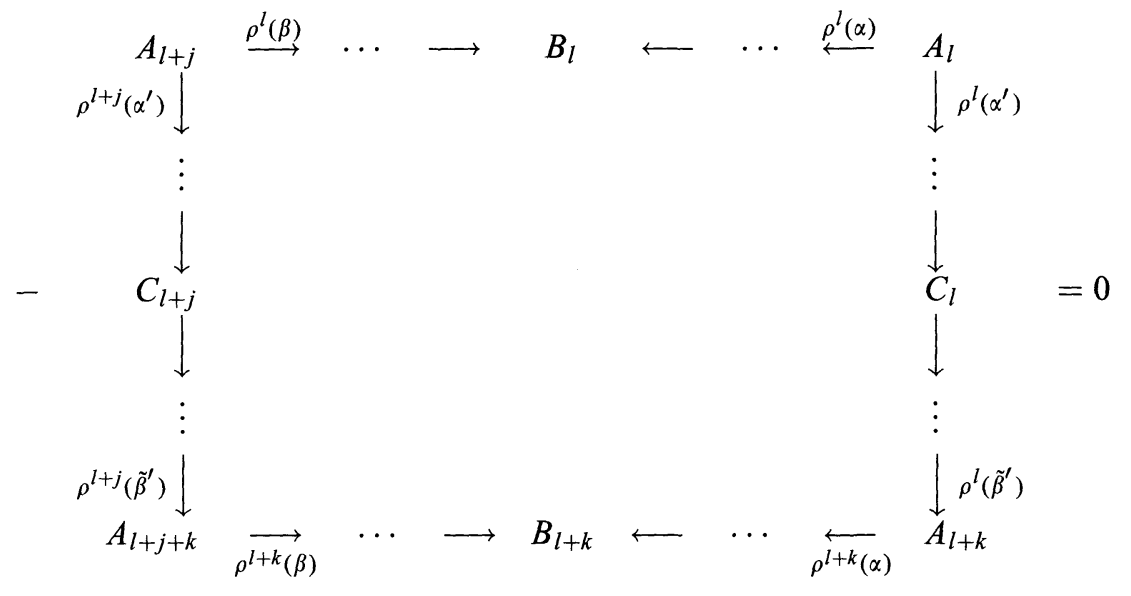

By Lemma 4.2, the values of the first and second terms in this expression are equal to 1 . Then equality (4.2) is equivalent to the following identity:

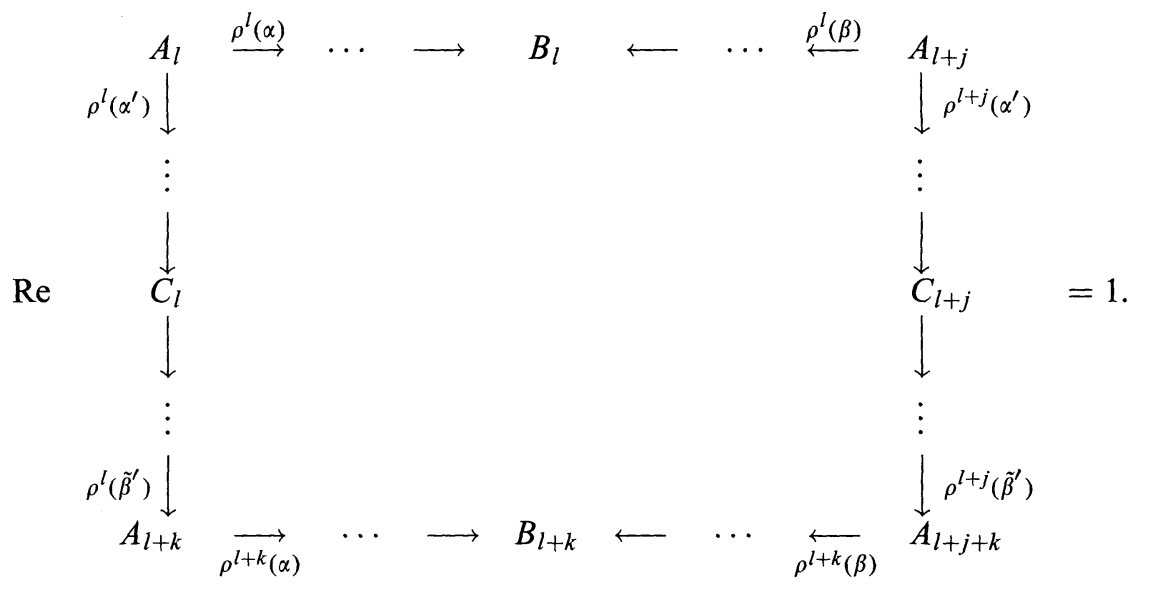

We will show that above equality (4.3) holds under the following assumptions.

Assumption 4.3.

(1) $N$ is odd.

(2) The graph $\mathscr{A}_{0}^{(n)}$ has no non-trivial graph automorphism fixing *.

Note that the above two conditions hold if $N=3$ and $n \geqq 9$. (See Fig. 3.1.) (When $N=3$ and $n=6$, we get a subfactor with index 4 , and classification of subfactors with index 4 is already known by [P4] (see also [IK]), we do not have to worry about this case.)

We make some comments on the case $N=2$. In this case, we only had to work on a single diagram as in [ $\mathrm{Ka}$, Lemma 5.2]. The original graph is $A_{n-1}$ and the orbifold graph is $D_{n / 2+1}$. It was proved in [Ka, Proposition 4.2] that the left-hand side of $(4.3)$ is 1 if $n \equiv 2(\bmod 4)$ and -1 if $n \equiv 0(\bmod 4)$. Thus we had flatness only in the case $n \equiv 2(\bmod 4)$. This means that a $\mathbf{Z} / 2 \mathbf{Z}$-obstruction for flatness appeared in the orbifold process. What we will prove here is that this obstruction vanishes under Assumption 4.3. 
Asuming that (4.3) is valid, we can compute the principal graph of the subfactor as the orbifold graph of $\mathscr{A}_{0}^{(n)}$ under $\rho$. Some examples are shown below for the case $N=3$.
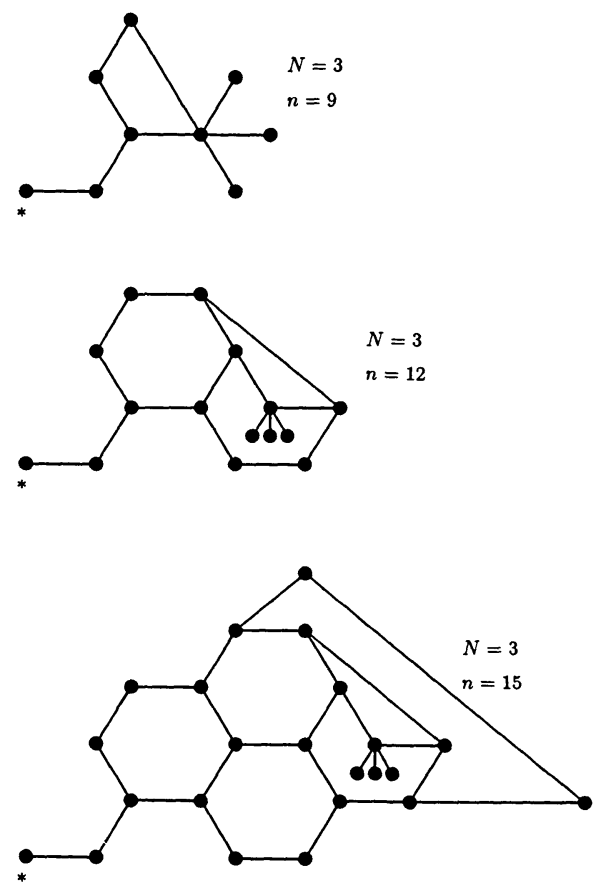

Fig. 4.3

Our proof of equality (4.3) is rather lengthy, so it is divided into the following steps. First set

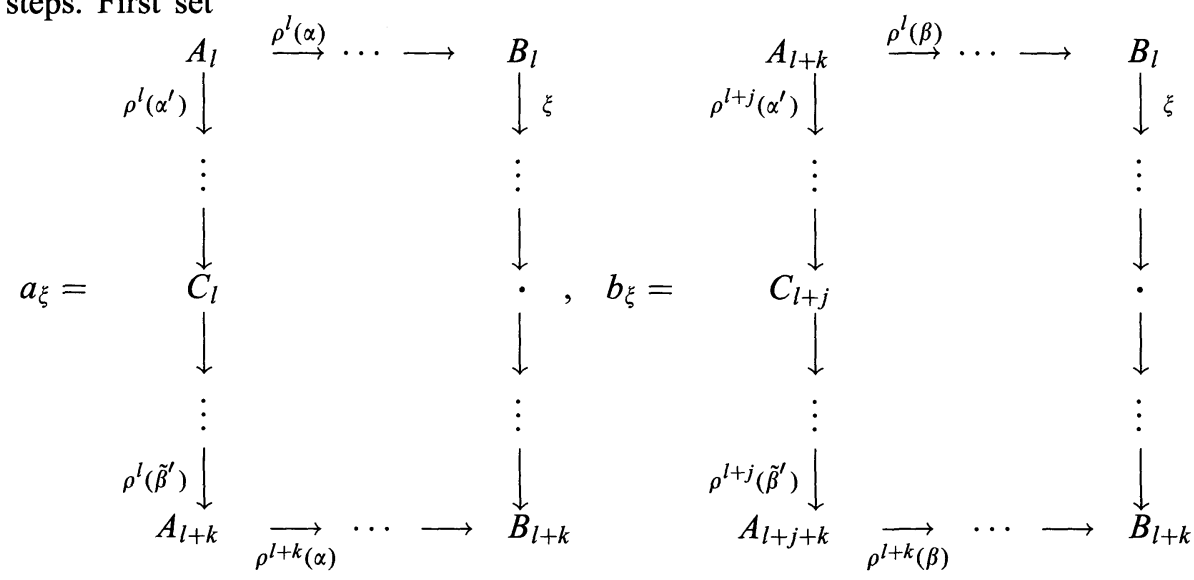

Denoting the partition function which appears in the left-hand side of (4.3) by $c$, we get $c=\sum_{\xi} a_{\xi} \bar{b}_{\xi}$. We prove $c=1$ under Assumption 4.3 in the following steps:

(1) Prove $\sum_{\xi}\left|a_{\xi}\right|^{2}=\sum_{\xi}\left|b_{\xi}\right|^{2}=1$ using the Yang-Baxter equation.

(2) Prove $\left|\sum_{\xi} a_{\xi} \bar{b}_{\xi}\right|=1$ using (2) of Assumption 4.3 and properties of the 
Jones projections. (This implies $a_{\xi}=c b_{\xi}$ for all $\xi$ and $|c|=1$ because we have equality in the Cauchy-Schwarz inequality.)

(3) Using Proposition 1.4, prove $c \in \mathbf{R}$, which means $c= \pm 1$.

(4) Prove $c=1$ if $B_{l}=C$, the fixed point of $\rho$, using (1) of Assumption 3.2.

(5) Get a contradiction from (4) by assuming $c=-1$ for some general case, where $B_{l}$ is arbitrary.

The first step is done by Lemma 4.2. Details of the other four steps will be given in the next section. Note that an induction as in [ $\mathrm{Ka}$, Proposition 4.2] does not work well in our situation because the graph is more complicated than that for $N=2$, the Dynkin diagrams $A_{n}$.

\section{Computations of Partition Functions}

We will prove $c=1$ under Assumption 4.3 in this section. First we need the following lemma.

Lemma 5.1. Let $\mathscr{G}$ be a bipartite unoriented graph with the distinguished vertex *. Denote the string algebra of strings with length $k$ with the starting point * by $\operatorname{String}_{*}^{(k)} \mathscr{G}$. Let $\pi$ be an automorphism of the string algebra, $\pi\left(\operatorname{String}_{*}^{(k)} \mathscr{G}\right)=$ String $_{*}^{(k)} \mathscr{G}$, with $\pi\left(e_{k}\right)=e_{k}$ for all the vertical Jones projections $e_{k}$ 's. If $\mathscr{G}$ has only single edges and $\mathscr{G}$ has no-nontrivial graph automorphism fixing $*$, then $\pi(\xi, \xi)=$ $(\xi, \xi)$ for all paths $\xi$.

Proof. Because the automorphism $\pi$ fixes the Jones projections, it gives an automorphism of the graph $\mathscr{G}$. By assumption, this graph automorphism is trivial.

We prove the assertion by induction on length of $\xi$. Suppose that we have $\pi(\xi, \xi)=(\xi, \xi)$ for $|\xi| \leqq k$ and we work for $\xi \cdot \alpha$ with $|\xi|=k,|\alpha|=1$.

Suppose first that $(\xi \cdot \alpha, \xi \cdot \alpha) \in \operatorname{String}_{*}^{(k)} \mathscr{G} \cdot e_{k} \cdot \operatorname{String}_{*}^{(k)} \mathscr{G}$. Then the string $(\xi \cdot \alpha, \xi \cdot \alpha)$ can be expressed as $a(\xi, \eta) e_{k}(\eta, \xi)$, where $|\eta|=k, a \in \mathbf{C}, a \neq 0$. By assumption, $\pi(\xi, \eta)=b(\xi, \eta)$ for some $b \in \mathbf{C},|b|=1$, thus we get $\pi(\xi, \xi)=(\xi, \xi)$.

Next assume that $(\xi \cdot \alpha, \xi \cdot \alpha) \notin \operatorname{String}_{*}^{(k)} \mathscr{G} \cdot e_{k} \cdot \operatorname{String}_{*}^{(k)} \mathscr{G}$. In this case we get $\pi(\xi, \xi)=(\xi, \xi)$ because the graph has only single edges and the induced graph automorphism is trivial.

Q.E.D.

\section{Lemma 5.2.}

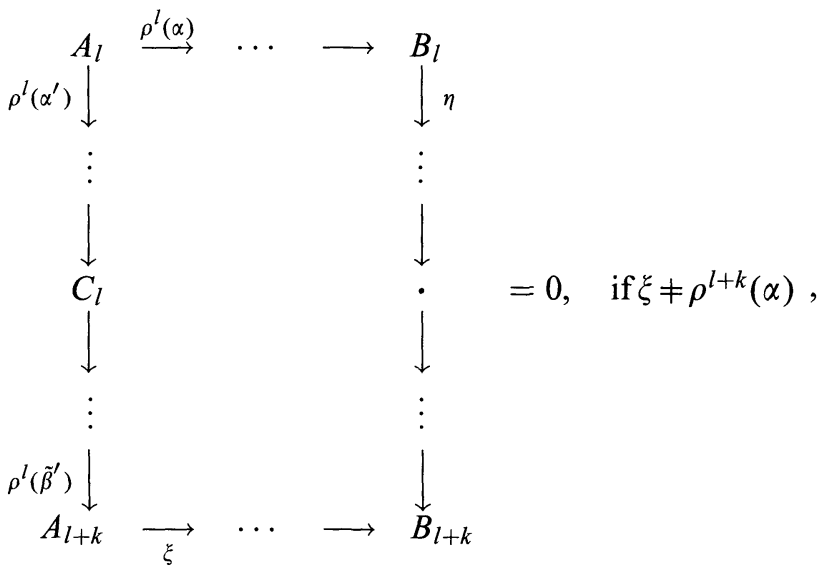


Proof. This easily follows from Lemma 4.2.

Q.E.D

\section{Lemma 5.3.}
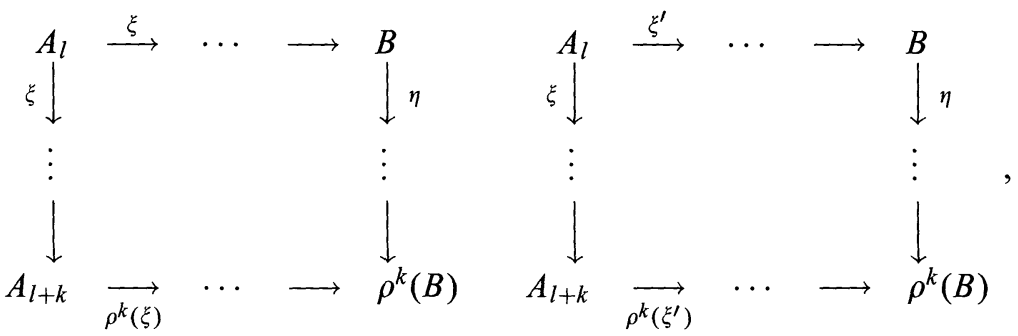

where $B$ is some vertex of $\mathscr{A}^{(n)}$ and $|\xi|=\left|\xi^{\prime}\right|$.

Proof. We make a sum over $\eta$ of the squares of the difference between the both hand sides as in Sect. 4. By Lemma 4.2, we get the sum is equal to $1+1-$ $1-1=0$.

Q.E.D.

The next Lemma proves step (2) at the end of Sect. 4.

Lemma 5.4. We have $|c|=1$.

Proof. First note that we have the following identity by Lemma 5.2:

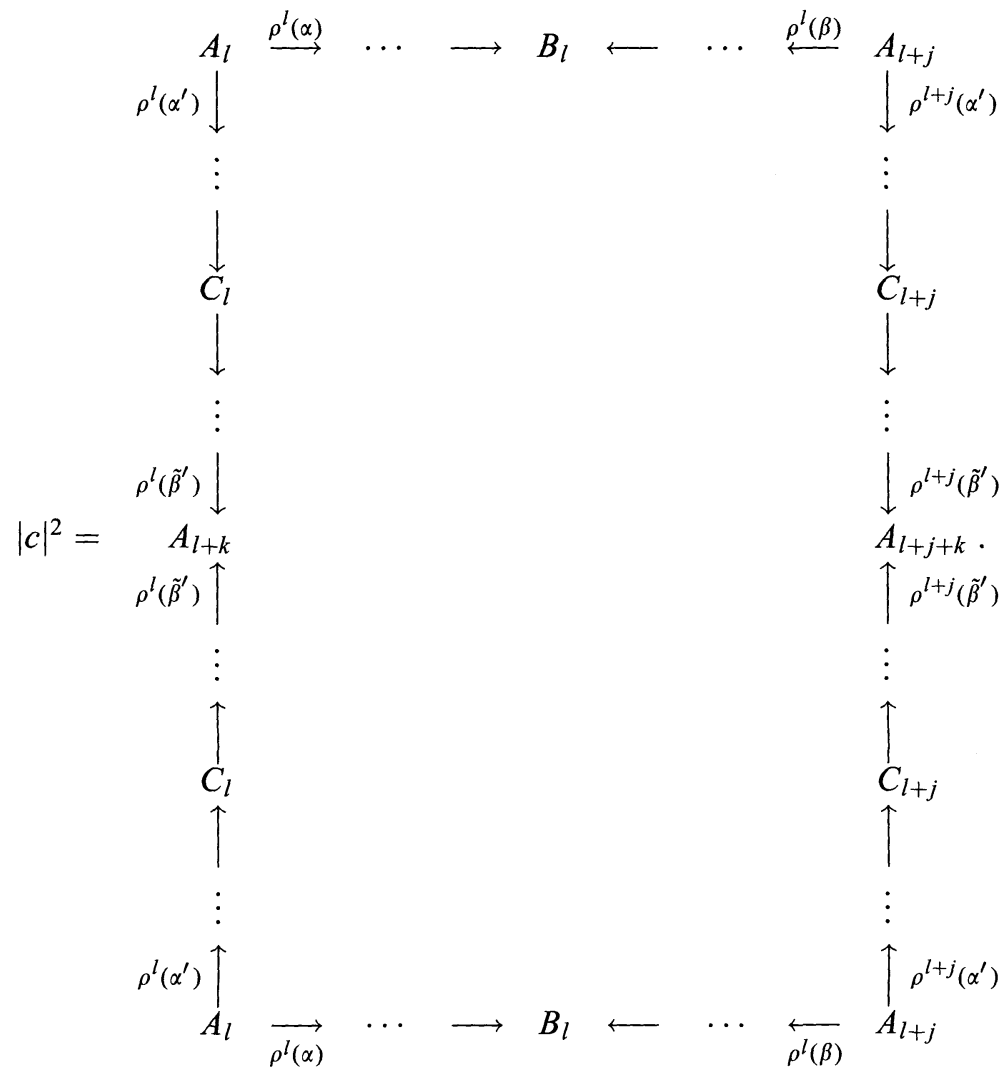


By applying Lemma 4.2 to the horizontal parallel transport from $A_{l}$ to $A_{l}$, we get the following.
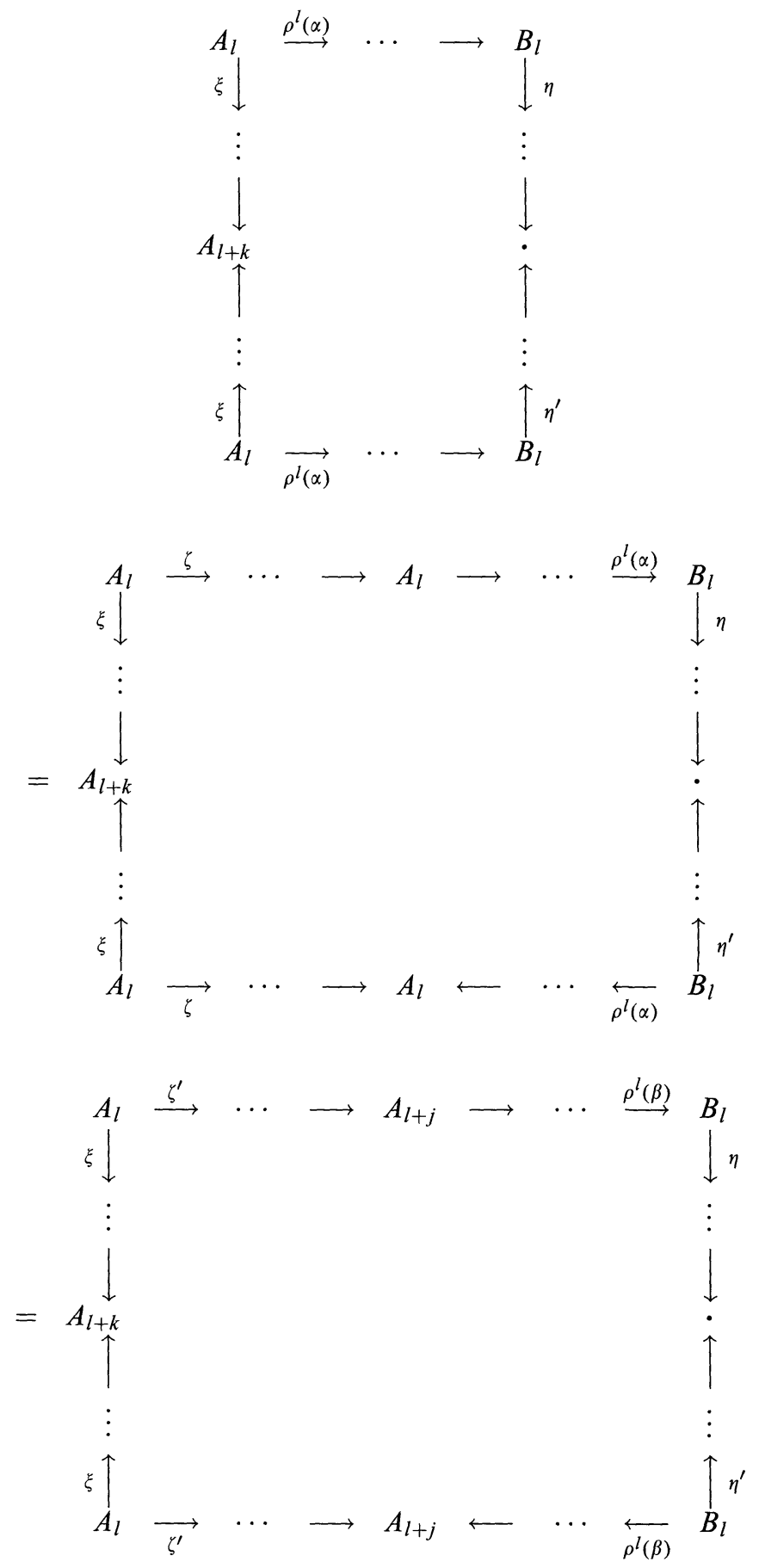


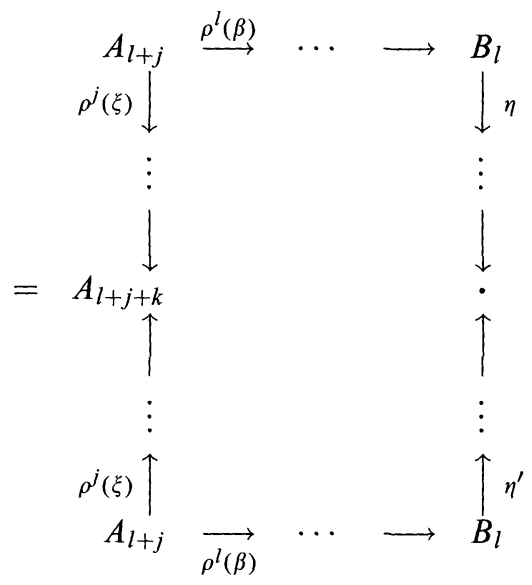

Here $|\xi|=\left|\xi^{\prime}\right|$ and we used Lemma 5.3 in the second equality. By this, we get

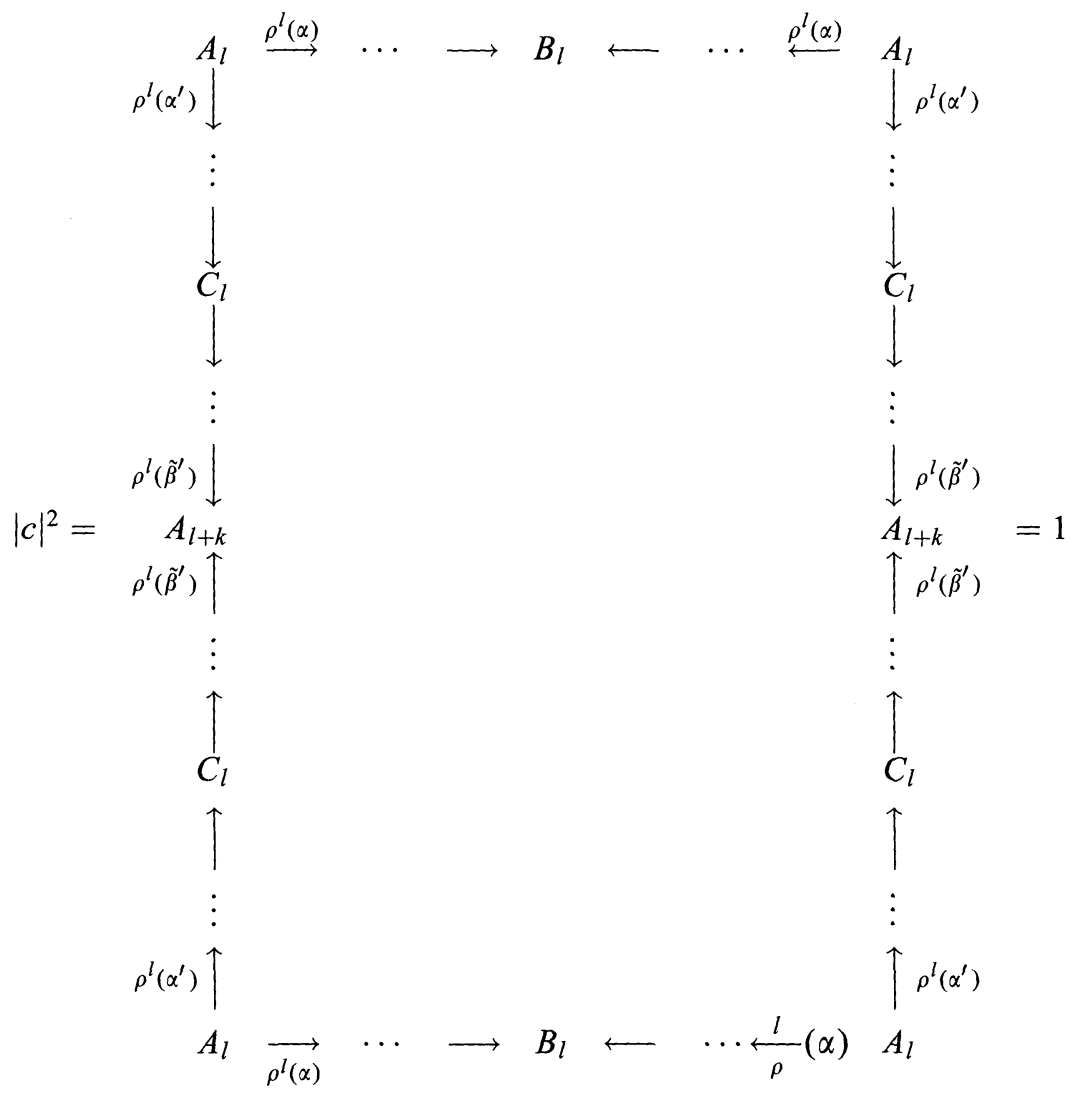

by Lemma 4.2 .

Q.E.D.

Now we have $a_{\xi}=c b_{\xi}$ for all $\xi$ by the Cauchy-Schwarz inequality. Next we claim that $c$ is real for the step (3).

Lemma 5.5 We get $c \in \mathbf{R}$. 
Proof. Choose $\xi$ so that $a_{\xi} \neq 0$. By Proposition 1.4,

$$
c \in \mathbf{R} \cdot \varepsilon^{A_{l} \cdot A_{l}+A_{l+j+k} \cdot A_{l+j+k}-A_{l+k} \cdot A_{l+k}-A_{l+j} \cdot A_{l+j} .}
$$

Choose $\eta_{1}, \eta_{2}, \zeta_{1}, \zeta_{2}$ so that

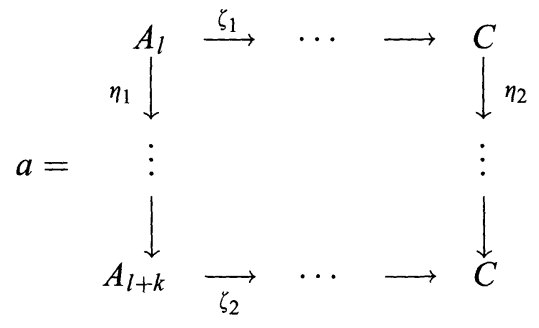

is non-zero. We also set

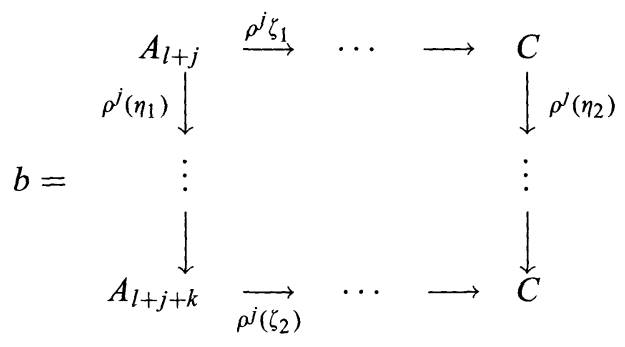

By invariance of the connections under $\rho$, we get $a=b$. On the other hand, if we apply the above arguments to $a$ and $b$, we get

$$
1=\frac{a}{b} \in \mathbf{R} \cdot \varepsilon^{A_{l}} \cdot A_{l}+A_{l+j+k} \cdot A_{l+j+k}-A_{l+k} \cdot A_{l+k}-A_{l+j} \cdot A_{l+J} .
$$

This implies $c \in \mathbf{R}$.

Q.E.D.

Next we have to determine the sign of $c$. First we assume that $B_{l}=C$ for all $l$.

Lemma 5.6. We get $c=1$ if $B_{l}=C$ for all $l$.

Proof. We have $a_{\xi}=c b_{\xi}$, but the fact that the connections are invariant under $\rho$ and Lemma 5.3 imply $b_{\xi}=a_{\rho^{-j}(\xi)}$. This produces

$$
a_{\xi}=c a_{\rho^{-j}(\xi)}=c^{2} a_{\rho^{-2 j}(\xi)}=\cdots=c^{N} a_{\rho^{-N j}(\xi)}=c^{N} a_{\xi}
$$

Choosing $\xi$ so that $a_{\xi} \neq 0$, we get $c^{N}=1$. Now $N$ is odd by (1) of Assumption 4.3 and $c$ is real by Lemma 5.5, thus we get $c=1$.

Q.E.D.

Finally we show $c=1$, without assuming that $B_{l}$ is the fixed point of $\rho$.

Lemma 5.7. We get $c=1$.

Proof. Suppose 


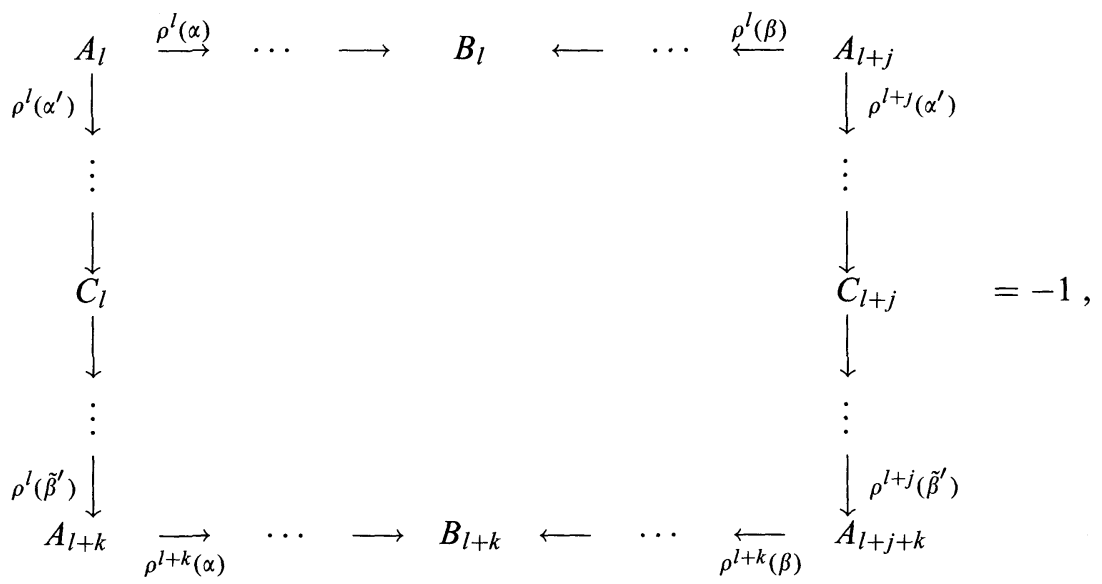

for some $l, k, j, \alpha, \beta, \alpha^{\prime}, \beta^{\prime}$. We will derive a contradiction.

Choose a positive integer $p$ large enough so that there exists a path from $B_{l}$ to $C$ with length $p$. Set

$$
K=\left\{\gamma\left|s(\gamma)=B_{l},\right| \gamma \mid=p\right\} \neq \varnothing
$$

Consider the following quantity.

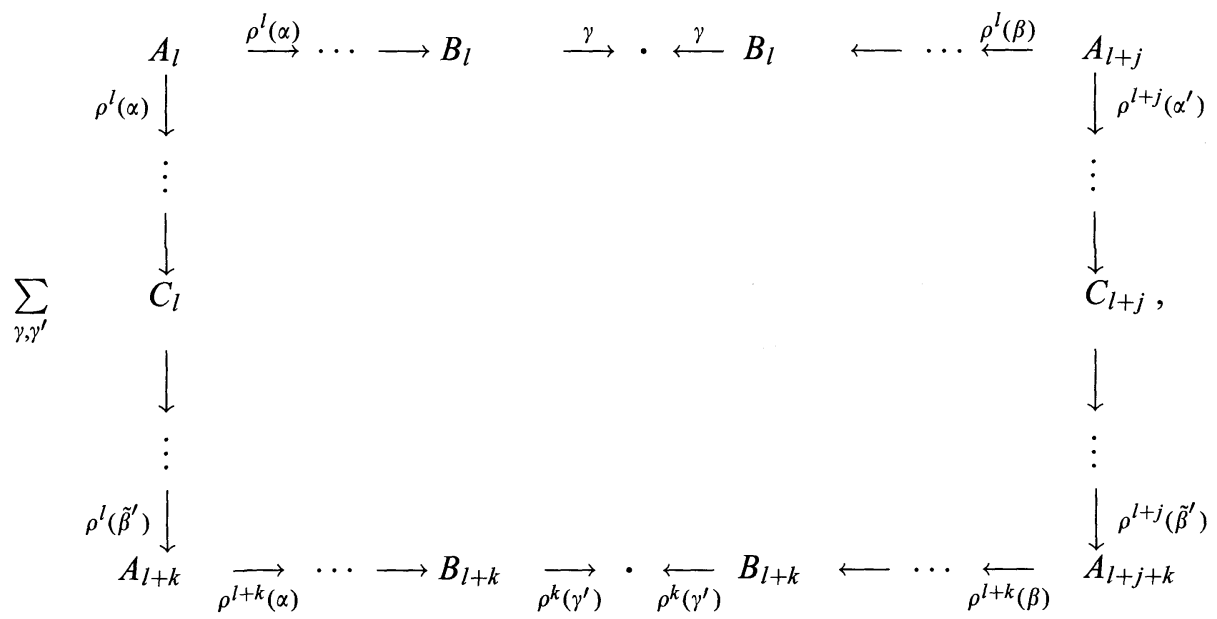

where $\gamma, \gamma^{\prime}$ are taken from the set $K$. We compute the value (4.1) in two ways.

On one hand, we take a sum in the way $\sum_{\gamma} \sum_{\gamma}^{\prime}$, then the value is $\sum_{\gamma}(-1)=$ $-|K|$ by unitarity of the connections.

On the other hand, we get $\gamma^{\prime}=\gamma$ if the diagram has non-zero value, by Lemma 5.2. Thus the value of $(5.1)$ is equal to 


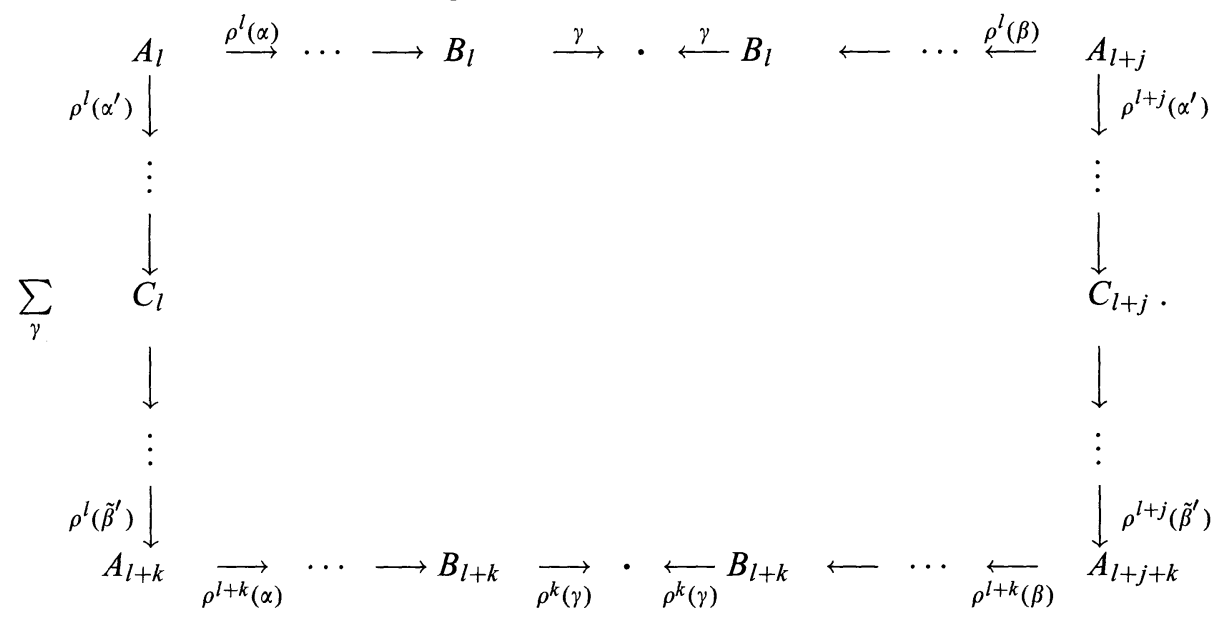

We have $|K|$ terms here and each term is \pm 1 by Lemmas 5.4 and 5.5 . But the sum must be $-|K|$, which means that all the terms must be -1 . But among these $|K|$ terms, there exists a term for which $r(\gamma)=C$ by definition of $p$. For such a term, the value must be 1 by Lemma 5.6. This is a contradiction.

Q.E.D.

Thus we have proved the following.

Theorem 5.8. The orbifold subfactor $D_{0, \infty} \subset D_{1, \infty}$ defined in Sect. 4 has the higher relative commutants $\left(D_{k, 0}\right)_{k}$ under Assumption 4.3 .

We end with three remarks.

Remark 5.9. For automorphisms of a factor fixing a subfactor globally, P. Loi introduced an invariant in [Li1], which is an action of the extended automorphism on the higher relative commutants. He showed that an outer action of $\mathbf{Z}$ on an AFD subfactor of type $\mathrm{II}_{1}$ with finite index and finite depth with his invariant trivial is unique up to outer conjugacy. The result of the second author in [Ka] shows that this kind of uniqueness fails for $\mathbf{Z}_{2}$ actions on subfactors of type $A_{4 n-3}$ because Loi's invariant is always trivial for subfactors of type $A_{n}$. Furthermore, we can prove that our $\mathbf{Z}_{3}$ action above also has Loi's invariant trivial. Indeed, Equality (4.3) gives that the higher relative commutants for the subfactor $C_{0, \infty} \subset C_{1, \infty}$ in the double sequence at the beginning of Sect. 4 is spanned by the elements $\sum_{k=0}^{N} \rho^{k}(\sigma)$, where $\sigma$ is any string with the starting vertex $A_{0}$. The extended action of our $\rho$ on the Jones tower is exactly the same as our original $\rho$, so it acts trivially on the higher relative commutants. So our action changes (actually increases) the higher relative commutants by making the simultaneous fixed point algebras (or crossed product algebras) while it has Loi's invariant trivial.

Remark 5.10. In [Li2, Lemma 4.2], P. Loi considered a symmetry of finite order of a paragroup and a fixed point algebra under it and noticed that the higher relative commutants are computed easily. In his work arising from a classification of subfactors of type $\mathrm{III}_{\lambda}$, he considers only symmetries fixing $*$ of the paragroup. But then a new paragroup obtained as a fixed point algebra has a symmetry moving $*$. Our symmetry for paragroups of the Hecke algebra subfactors of Wenzl moves $*$. Thus our construction here can be regarded as the inverse direction of Loi's work. 
This inverse direction is more interesting and difficult because the flatness is not automatically satisfied.

Remark 5.11. So far, we have been unable to prove that (2) of Assumption 4.3 holds for odd $N$ in general with some rather trivial exceptions. But with slight modification of the above arguments, we can prove flatness for the orbifold construction for general odd prime $N$ as follows.

In the construction of the double sequence $C_{j k}$, we used period $N$ commuting square sequences in the horizontal direction and period 2 sequences in the vertical direction. But we have computed the paragroups for the Hecke algebra subfactors of Wenzl in Theorem 3.5, so we can use the period 2 sequences in both directions for the double sequence $C_{j k}$. Then we lose the Yang-Baxter equation, but we have a flatness for the original paragroup, so the following weaker version of Assumption 4.3 (2) is enough for the arguments in Sect. 4.

$(2)^{\prime}$ The graph $\mathscr{A}_{0}^{(n)}$ has no non-trivial graph automorphism with order $N$ fixing $*$.

If $N$ is odd prime, then one can easily prove this $(2)^{\prime}$ by induction, and we get flatness for the orbifold construction in these cases, which produces several new series of orbifold subfactors.

\section{References}

[ABF] Andrews, G.E., Baxter, R.J., Forrester, P.J.: Eight vertex SOS model and generalized Rogers-Ramanujan type identities. J. Stat. Phys. 35, 193-266 (1984)

[Ba] Baxter, R.J.: Exactly solved models in statistical mechanics, New York: Academic Press,. 1982

[Bi] Bisch, D.: On the existence of central sequences in subfactors. Trans. Am. Math. Soc. 321, 117-128 (1990)

[Bl] Blackadar, B.: Symmetries of the CAR algebra, Ann. Math. 131, 589-623 (1990)

[BEEK1] Bratteli, O., Elliott, G.A., Evans, D.E., Kishimoto, A.: Non-commutative spheres. Inter. J. Math. 2, 139-166 (1991)

[BEEK2] Bratteli, O., Elliott, G.A., Evans, D.E., Kishimoto, A.: Finite group actions on AF algebras obtained by folding the interval. (To appear in K-theory)

[BEEK3] Bratteli, O., Elliott, G.A., Evans, D.E., Kishimoto, A.: Non-commutative spheres II: Rational rotations. 27, 53-85 (1992) J. Operator Theory

[BEK] Bratteli, O., Evans, D.E., Kishimoto, A.: Crossed products of totally disconnected spaces by $\mathbf{Z}_{2} * \mathbf{Z}_{2}$. Ergodic Th. Dyn. Sys. 13, 445-484 (1993)

[BK] Bratteli, O., Kishimoto, A.: Non-commutative spheres III: Irrational rotations. Commun. Math. Phys. 147, 605-624 (1992)

[C] Choda, M.: Duality for finite bipartite graphs. Pac. J. Math. 158, 49-65 (1993)

[CE] Connes, A., Evans, D.E.: Embeddings of $U(1)$-current algebras in non-commutative algebras of classical statistical mechanics. Commun. Math. Phys. 121, 507-525 (1989)

[DJMO] Date, E., Jimbo, M., Miwa, T., Okado, M.: Solvable lattice models. In: Theta functions - Bowdoin 1987, Part 1. Proc. Sympos. Pure Math. Vol. 49, Providence, RI: Am. Math. Soc., pp. 295-332

[DZ1] Di Francesco, P., Zuber, J.-B.: $S U(N)$ lattice integrable models associated with graphs. Nucl. Phys. B338, 602-646 (1990)

[DZ2] Di Francesco, P., Zuber, J.-B.: $S U(N)$ lattice integrable models and modular invariance. Nucl. Phys. (Proc. Suppl.) B18, 313 (1990)

[DHVW] Dixon, L., Harvey, J.A., Vafa, C., Witten, E.: Strings on orbifolds. Nucl. Phys. B261, 678-686 (1985), B274, 285-314 (1986)

[Ell] Elliott, G.A.: A classification of certain simple $C^{*}$-algebras. In: Quantum and noncommutative analysis, Kluwerv Academic, 1993, pp. 373-386. 
[EE] Elliott, G.A., Evans, D.E.: The structure of the irrational rotation $C^{*}$-algebra. Ann. Math. 138, 477-501 (1993)

[E1] Evans, D.E.: The $C^{*}$-algebras of topological Markov chains. Tokyo Metropolitan University Lecture Notes, 1984

[E2] Evans, D.E.: Quasi-product states on $C^{*}$-algebras. In: "Operator algebras and their connections with topology and ergodic theory." Springer Lecture Notes in Math. 1132, 129-151 (1985)

[EG1] Evans, D.E., Gould, J.D.: Dimension groups and embeddings of graph algebras. to appear in Int. J. Math.

[EG2] Evans, D.E., Gould, J.D.: Presentations of AF algebras associated to T-graphs, to appear in Publ. RIMS, Kyoto Univ.

[EL] Evans, D.E., Lewis, J.T.: On a $C^{*}$-algebra approach to phase transition in the twodimensional Ising model II. Commun. Math. Phys. 102, 521-535 (1986)

[F] Fendley, P.: New exactly solvable orbifold models. J. Phys. A 22, 4633-4642 (1989)

[FG] Fendley, P., Ginsparg, P.: Non-critical orbifolds. Nucl. Phys. B324, 549-580 (1989)

[FYHLMO] Freyd, P., Yetter, D., Hoste, J., Lickorish, W., Millet, K., Ocneanu, A.: A new polynomial invariant of knots and links. Bull. Am. Math. Soc. 12, 239-246 (1985)

[GHJ] Goodman, F., de la Harpe, P., Jones, V.F.R.: Coxeter graphs and towers of algebras. MSRI publications 14, Berlin; Heidelberg, New York: Springer, 1989

[GW] Goodman, F., Wenzl, H.: Littlewood-Richardson coefficients for the Hecke for the Hecke algebras at roots of unity. Adv. Math. 82, 244-265 (1990)

[HS] Haagerup, U., Schou, J.: In preparation

[I] Izumi, M.: Application of fusion rules to classification of subfactors. Publ. RIMS Kyoto Univ. 27, 953-994 (1991)

[IK] Izumi, M., Kawahigashi, Y.: Classification of subfactors with the principal graph $D_{n}^{(1)}$. J. Funct. Anal. 112, 257-286 (1993)

[Ji] Jimbo, M. (ed.): Yang-Baxter equation in integrable systems. Advanced Series in Mathematical Physics, Vol. 10, Singapore: World Scientific, 1989

[JMO1] Jimbo, M., Miwa, T., Okado, M.: Solvable lattice models whose states are dominant integral weights of $A_{n-1}^{(1)}$. Lett. Math. Phys. 14, 123-131 (1987)

[JMO2] Jimbo, M., Miwa, T., Okado, M.: Solvable lattice models related to the vector representation of classical simple Lie algebras. Commun. Math. Phys. 116, 507-525 (1988)

[Jo] Jones, V.F.R.: Index for subfactors. Invent. Math. 72, 1-15 (1983)

[Ka] Kawahigashi, Y.: On flatness of Ocneanu's connections on the Dynkin diagrams and classification of subfactors. to appear in J. Funct. Anal.

[Kn] Kohno, T. (ed.): New developments in the theory of knots. Advanced Series in Mathematical Physics, Vol. 11, Singapore: World Scientific, 1989

[Ko] Kostov, I.: Free field presentation of the $A_{n}$ coset models on the torus. Nucl. Phys. B300, 559-587 (1988)

[Ku] Kumjian, A.: An involutive automorphism of the Bunce-Deddens algebra. CR Math. Rep. Acad. Sci. Canada 10, 217-218 (1988)

[Li1] Loi, P.H.: On automorphisms of subfactors. Preprint, UCLA, 1990

[Li2] Loi, P.H.: On the derived tower of certain inclusions of type $\mathrm{III}_{\lambda}$ factors of index 4 . to appear in Pac. J. Math.

[Ln1] Longo, R.: Index of subfactors and statistics of quantum fields I. Commun. Math. Phys. 126, 217-247 (1989)

[Ln2] Longo, R.: Index of subfactors and statistics of quantum fields II. Commun. Math. Phys. 130, 285-309 (1990)

[M] Murakami, J.: The representations of the $q$-analogue of Brauer's centralizer algebras and the Kauffman polynomial of links. Publ. RIMS Kyoto Univ. 26, 935-945 (1990)

[O1] Ocneanu, A.: Quantized group string algebras and Galois theory for algebras. In: Operator algebras and applications, Vol. 2 (Warwick, 1987). London Math. Soc. Lect. Note Series Vol. 136, Cambridge: Cambridge University Press, 1988, pp. 119-172

[O2] Ocneanu, A.: Graph geometry, quantized groups and nonamenable subfactors. Lake Tahoe Lectures, June-July, 1989 
[03] Ocneanu, A.: Quantum symmetry, differential geometry of finite graphs and classification of subfactors. University of Tokyo Seminary Notes 45, (Notes recorded by Y. Kawahigashi), 1991

[Ok] Okamoto, S.: Invariants for subfactors arising from Coxeter graphs. In: Current Topics in Operator Algebras. Singapore: World Scientific Publishing, 1991, pp. 84-103

[Pa] Pasquier, V.: Two-dimensional critical systems labelled by Dynkin diagrams. Nucl. Phys. B285, 162-172 (1987)

[PP] Pimsner, M., Popa, S.: Iterating the basic constructions. Trans. Am. Math. Soc. 310, 127-134 (1988)

[P1] Popa, S.: Orthogonal pairs of $*$-subalgebras in finite von Neumann algebras. J. Operator Theory 9, 253-268 (1983)

[P2] Popa, S.: Relative dimension, towers of projections and commuting squares of subfactors. Pac. J. Math. 137, 181-207 (1989)

[P3] Popa, S.: Classification of subfactors: Reduction to commuting squares. Invent. Math. 101, 19-43 (1990)

[P4] Popa, S.: Sur la classification des sousfacteurs d'indice fini du facteur hyperfini. C. R. Acad. Sc. Paris. 311, 95-100 (1990)

[R] Roche, Ph.: Ocneanu cell calculus and integrable lattice models. Commun. Math. Phys. 127, 395-424 (1990)

[Sc] Schou, J.: Commuting squares and index for subfactors. Ph. D. Thesis, Odense University, 1990

[So] Sochen, N.: Integrable models through representations of the Hecke algebra. Nucl. Phys. B360, 613-640 (1991)

[Su] Sunder, V.S.: A model for AF-algebras and a representation of the Jones projections. J. Operator Theory 18, 289-301 (1987)

[SV] Sunder, V.S., Vijayarajan, A.K.: On the non-occurrence of the Coxeter graphs $\mathrm{E}_{7}, D_{2 n+1}$ as principal graphs of an inclusion of $I_{1}$ factors. Pac. J. Math. 161, 185-200 (1993)

[Wa] Wassermann, A.J.: Coactions and Yang-Baxter equations for ergodic actions and subfactors. In: Operator algebras and applications, Vol. 2 (Warwick, 1987). London Math. Soc. Lect. Note Series Vol. 136, Cambridge Cambridge University Press, 1988, pp. 203-236

[We] Wenzl, H.: Hecke algebras of type A and subfactors. Invent. Math. 92, 345-383 (1988)

[YG] Yang, C.N., Ge, M.L. (eds.): Braid group, knot theory and statistical mechanics. Advanced Series in Mathematical Physics, Vol. 9, Singapore: World Scientific, 1989

[Z] Zuber, J.-B.: Graphs, algebras, conformal field theories and integrable lattice models. Nucl. Phys. (Proc. Suppl.) B18, 313 (1990)

Communicated by N. Yu. Reshetikhin

Note added in proof. After the submission of this paper, F. Xu computed our obstructions for flatness in all the cases by identifying them with conformal dimensions in the following papers.

[X1] F. Xu, Orbifold construction in subfactors, preprint 1992.

[X2] F. Xu, The flat parts of non-flat orbifolds, to appear in Pac. J. Math.

S. Goto also extended the orbifold constructions to subfactors without being AFD in the following papers.

[G1] S. Goto, Orbifold construction for non-AFD subfactors, to appear in Int. J. Math.

[G2] S. Goto, Symmetric flat connections, triviality of Loi's invariant and orbifold subfactors, preprint 1994. 